\title{
SURFACE MODIFICATION OF MICROPOROUS POLYPROPYLENE MEMBRANE BY UV-INITIATED GRAFTING WITH POLY(ETHYLENE GLYCOL) DIACRYLATE
}

\author{
A Thesis \\ Presented to the Faculty of the Graduate School \\ of Cornell University \\ In Partial Fulfillment of the Requirements for the Degree of \\ Master of Science
}

by

Mark Andrew Wing-Ki Chan

August 2012 
(C) 2012 Mark Andrew Wing-Ki Chan 


\begin{abstract}
In this study, poly(ethylene glycol) diacrylate (PEGDA) was surface grafted, through UV-initiated grafting, on to a microporous polypropylene (PP) membrane in order to develop and control a moisture-sensitive porous structure. Based on the concentration of the PEGDA grafting solution, as well as other variables, the pores of the membrane were filled to varying degrees with cross-linked PEGDA hydrogel, decreasing the pore sizes. This decrease in pore size was highly dependent on the grafting degree, or weight add-on of the grafted polymer. The grafting degree can be controlled by altering various grafting conditions. The surface grafted PEGDA is expected to swell significantly when exposed to moisture, through change in relative humidity or a liquid-borne pathogen, causing the pore sizes to decrease even further. This provides a microporous polypropylene membrane with improved hydrophilicity and moisture-responsive pores. The membranes will have varying levels of breathability based on the amount of moisture exposure. This will allow for a functional membrane that limits the transport of liquid-borne pathogens while providing transport of moisture vapor away from the body.
\end{abstract}




\section{BIOGRAPHICAL SKETCH}

Mark Chan was born in sunny California during the Chinese year of the Dragon, 1988. After a long and successful career from pre-school through high school, he decided to further his education at the University of California, Davis. There, under the tutelage of Professor You-Lo Hsieh, Mark graduated in 2010 as the top, and only, student for the Fiber and Polymer Science B.S. program in the department of Textiles and Clothing within the College of Agriculture at the University of California, Davis. From there, he decided to yet again further his education. Mark ventured forth, driving across the country and arriving in not-as-sunny New York state. There, he attended Cornell University in a valiant attempt to achieve a M.S. degree in Fiber Science from the Fiber Science \& Apparel Design department within the College of Human Ecology. Under the mentorship of Professor S. Kay Obendorf, Mark hopes to complete his degree in 2012 and set off once again to continue his unique journey, just like everyone else. 


\section{ACKNOWLEDGMENTS}

I would like to thank my advisor, Professor S. Kay Obendorf, for the wonderful support she gave me as I worked toward my degree. I would also like to thank my minor committee member, Professor Anil Netravali, for his support of my degree. My research group consisting of Laura Lange, Elizabeth Allen, Dong Jin Woo, and Yunfei Han have also greatly supported me through my education. I would like to thank the Fiber Science \& Apparel Design department members and faculty for their continued support. I also give thanks to the AATCC foundation for providing support to my project. 
TABLE OF CONTENTS

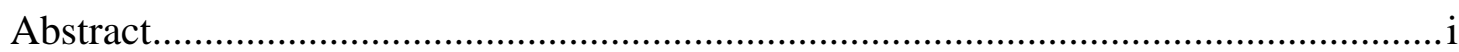

Biographical Sketch.................................................................................................iii

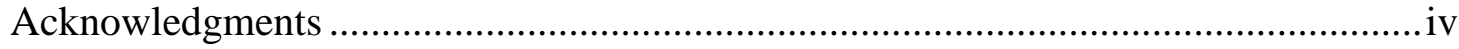

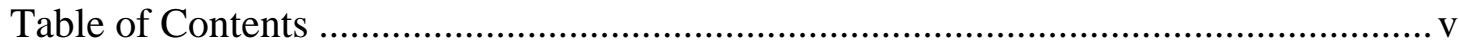

List of Figures........................................................................................................ vii

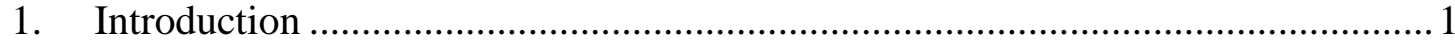

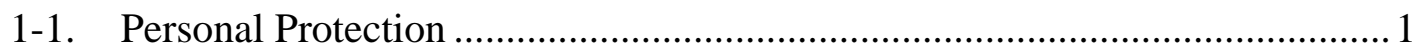

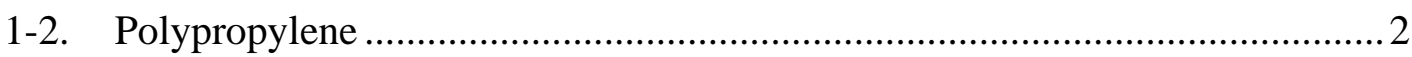

1-3. Poly(ethylene glycol) diacrylate ………………..............................................

1-4. Surface Modification and Grafting ............................................................... 5

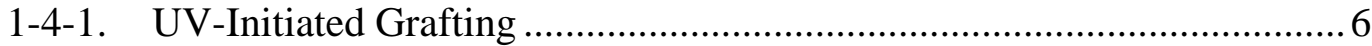

1-4-2. Plasma Grafting .............................................................................. 8

1-4-3. Ozone Grafting ............................................................................... 8

1-5. Grafting with Poly(ethylene glycol) ............................................................

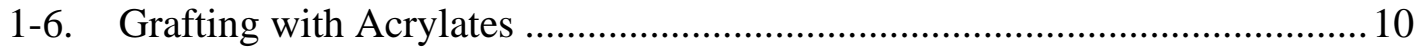

1-6-1. N,N-dimethylamino ethyl methacrylate ................................................... 10

1-6-2. D-gluconamidoethyl methacrylate ………………………..................... 12

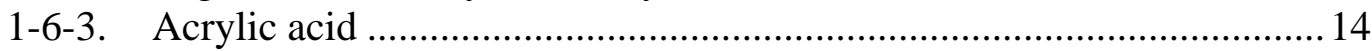

1-7. Grafting with Acrylated Poly(ethylene glycol) .............................................. 15

1-7-1. Plasma Grafting ................................................................................ 15

1-7-2. Ozone Grafting ................................................................................ 17

1-7-3. UV-Initiated Grafting for Responsive Macropores................................... 18

1-7-4. UV-Initiated Grafting on Poly(dimethylsiloxane).................................... 19

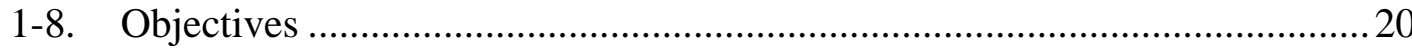

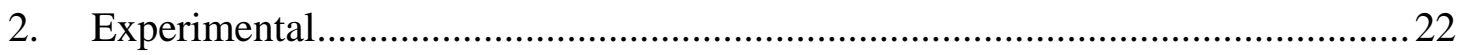

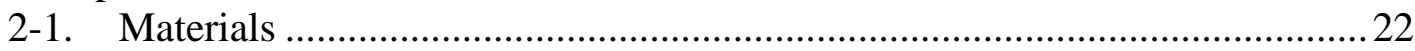

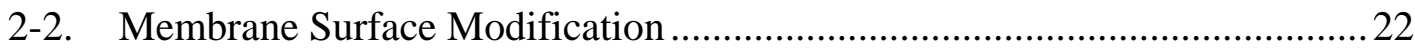

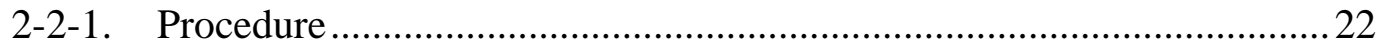

2-2-2. Proposed Grafting Scheme .................................................................... 24

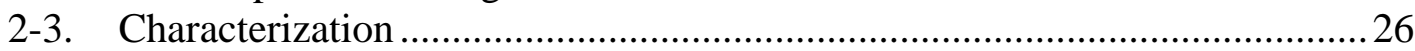

2-3-1. Grafting Conditions ............................................................................ 26

2-3-2. Surface Morphology ………………………………………………...... 26

2-3-3. Fourier Transform Infrared Spectroscopy ………………………….......26

2-3-4. Contact Angle Measurements................................................................. 28

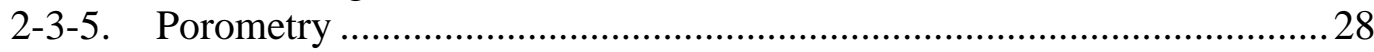

2-3-6. Statistical Analysis ................................................................................... 30 


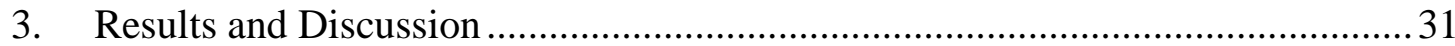

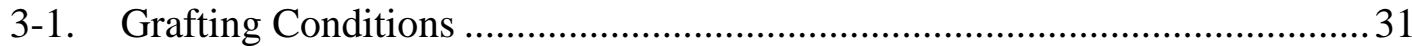

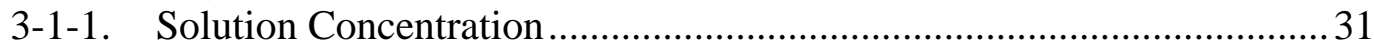

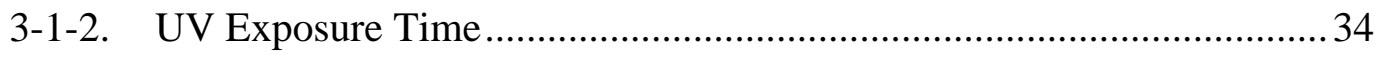

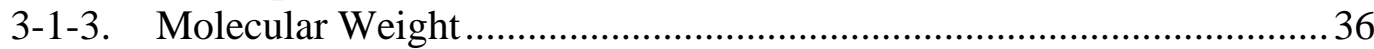

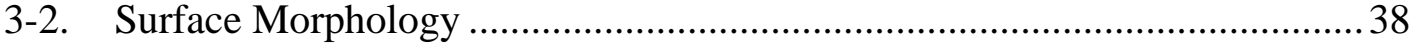

3-3. Fourier Transform Infrared Spectroscopy .................................................... 40

3-4. Contact Angle Measurements ........................................................... 42

3-5. Porometry .................................................................................... 44

3-5-1. Air Flow Rate Comparisons of Various Grafting Degrees ................... 44

3-5-2. Pore Size Distribution Comparisons of Various Grafting Degrees........ 48

3-5-3. Air Flow Rate Comparison of Different Relative Humidity .................. 50

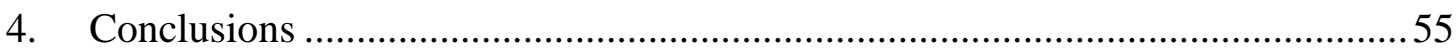

4-1. Recommendations for Future Studies..................................................... 56

4-1-1. Water Vapor Transmission Rate .......................................................56

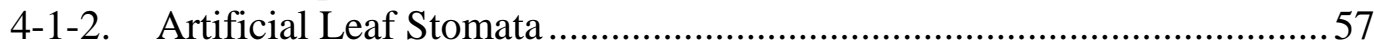

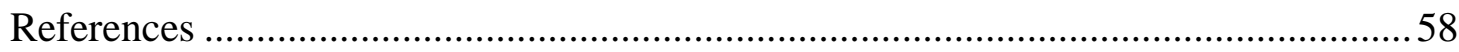




\section{LIST OF FIGURES}

Figure 1. PEGDA synthesis and cross-linking ................................................ 4

Figure 2. Mechanism of UV-initiated surface grafting with benzophenone as the photoinitiator and an inert substrate surface............................................. 7

Figure 3. Comparison of the monofunctionality of DMAEMA to the difunctionality of

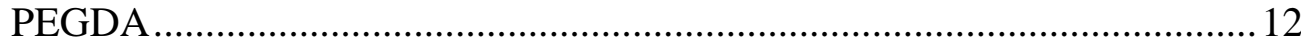

Figure 4. Plasma grafting of PEGDA on to membrane surface ................................ 16

Figure 5. Illustration of proposed schematic for the surface grafting of microporous polypropylene membrane's porous structure with PEGDA......................... 24

Figure 6. FT-IR spectra of PEG and PEGDA .................................................. 27

Figure 7. Capillary flow porometer output plot of an untreated microporous polypropylene membrane .................................................................... 30

Figure 8. The effect of PEGDA grafting solution concentration on grafting degree with PEGDA $\mathrm{M}_{\mathrm{n}} 575$ and 15 min of UV exposure .......................................... 31

Figure 9. The effect of UV exposure time on grafting degree with PEGDA $M_{n} 575$ and $5 \mathrm{wt} . \%$ grafting solution concentration ................................................... 34

Figure 10. The effect of PEGDA molecular weight on grafting degree with 5 wt.\% grafting solution concentration and 15 min of UV exposure ...................36

Figure 11. SEM images of PEGDA grafted microporous polypropylene membrane... 38

Figure 12. FTIR spectra of PEGDA grafted microporous polypropylene membrane .40

Figure 13. The effect of grafting degree on water contact angle of PEGDA grafted microporous polypropylene membranes ............................................. 42

Figure 14. Dry air flow rate comparisons of PEGDA grafted microporous polypropylene membranes with various grafting degrees 44

Figure 15. JMP statistical output for the ANCOVA of the dry air flow rate comparisons of membranes with various grafting degrees 46 
Figure 16. Pore size distributions of PEGDA grafted microporous polypropylene membranes with various grafting degrees

Figure 17. Dry air flow rate comparison of PEGDA grafted microporous polypropylene membranes conditioned at different relative humidity.......50

Figure 18. JMP statistical output for the ANCOVA of the dry air flow rate comparison of grafted membranes conditioned at different relative humidity ................52 


\section{CHAPTER 1}

\section{INTRODUCTION}

\section{1-1. Personal Protection}

Depending on the application for a particular textile, pore size may be very important for personal protection. A smaller pore size generally provides a greater degree of protection from harmful microorganisms. However, a smaller pore size also decreases the amount of water vapor transmission as well as air permeability of the material, decreasing the comfort of the user. In this study, a membrane which reduces in pore size when exposed to moisture, through change in relative humidity or a liquid-borne pathogen, will be created. This response to moisture can provide a greater degree of protection from liquid-borne pathogens while offering a reasonable degree of comfort to the user. The increased hydrophilicity of the hydrophobic polypropylene membrane will also help to improve various characteristics, such as antifouling properties [1].

Currently, much research on personal protective equipment (PPE) is focused on balancing comfort with protection. Generally, a greater degree of protection comes at the sacrifice of comfort. On one end of the spectrum, there are impermeable membranes which allow nothing, including water vapor and air, to pass through. This can be quite uncomfortable for a user, but can provide a high degree of protection. On the other end of the spectrum are untreated woven textiles, where high comfort is offered, but little protection. There have been many studies done in an attempt to find a balance between comfort and protection: self-decontaminating melt-spun 
polypropylene (PP) fibers for pesticide protection [2], antifungal properties on electrospun cellulose membranes [3], melt-electrospun PP fibers for pesticide protection [4]. This current study was conducted in an attempt to find a balance between comfort and protection through the use of microporous membranes which provide protection due to the relatively small pore sizes. However, since pore sizes exist in the membrane, there is still some protection sacrificed for comfort. This study attempts to create moisture-responsive pores within the microporous membrane which will respond to stimuli, such as liquid-borne pathogens, in order to enhance protection.

\section{1-2. Polypropylene}

Polypropylene is a thermoplastic polyolefin used in a large variety of applications. PP is known to be resistant against many solvents. Polypropylene can be created in many different forms such as plastics and membranes. In the case of PPE, the membrane form is often used. Microporous polypropylene membranes can be created in order to provide protection while offering some degree of comfort. The porosity of these membranes can be highly controlled in order to create a finely tuned membrane. PP is naturally hydrophobic, although modification to impart hydrophilicity is common. These properties of polypropylene make it a popular material for use in protective equipment [5]. 


\section{1-3. Poly(ethylene glycol) diacrylate}

Poly(ethylene glycol) (PEG) is a common polymer with a simple carbon chain structure with ether linkages. PEG is well known for its hydrophilicity, water solubility, and biocompatibility. PEG can be easily chemically modified or attached to the surfaces of other materials without greatly affecting the properties of PEG. PEG is nontoxic, can interact with cell membranes, and prevents protein adsorption, making it a popular polymer in the biomedical field. The prevention of protein adsorption is particularly important for achieving anti-fouling properties on a membrane surface. The hydroxyl end groups of PEG provide functional groups which allow covalent bonding to other materials [6]. These hydroxyl end groups can also be chemically replaced by acrylate groups in order to form poly(ethylene glycol) diacrylate (PEGDA). These acrylate groups can then be used to form covalent bonds with other PEGDA chains’ acrylate end groups in order to create a cross-linked network, yielding a hydrogel with interesting swelling properties [7]. 
<smiles>COCCOC(C)(C)CCO</smiles>

PEG<smiles>C=CC(=O)OCCOCCC(C)(C)OCCOC(=O)C=C</smiles>
PEGDA

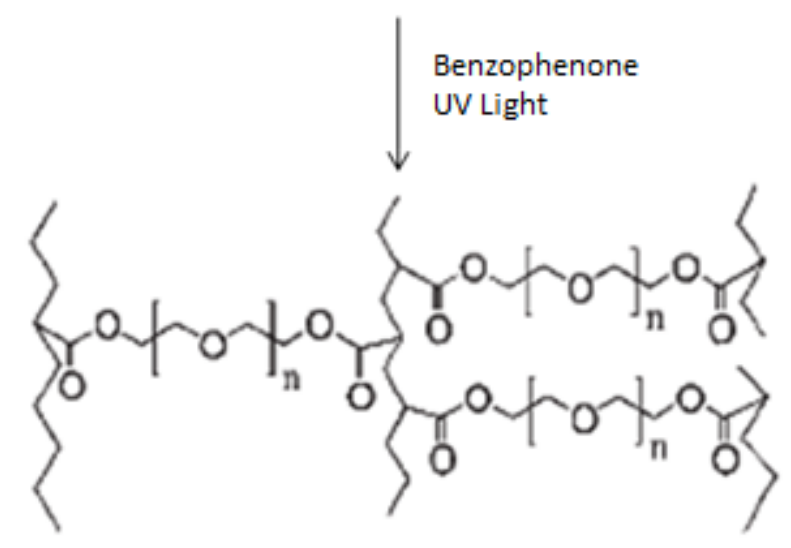

Cross-linked PEGDA

Figure 1. PEGDA synthesis and cross-linking [8]

Figure 1 is a schematic for the synthesis of PEGDA from PEG as well as the cross-linking of PEGDA to form a hydrogel. The synthesis of PEGDA involves the exchange of the acrylate group from excess acryloyl chloride under basic conditions provided by excess triethylamine. Insoluble triethanolamine hydrochloride is a byproduct which is filtered out. Then the PEGDA product is usually precipitated out 
of solution. PEGDA cross-linking involves exposure to UV light with a photo-initiator such as benzophenone. The carbon-carbon double bonds of the acrylate end groups are consumed in the reaction in order to form a hydrogel.

\section{1-4. Surface Modification and Grafting}

Surface modification of membranes is a common method for improving various properties of the nascent membrane. Improvements to a membrane's permeability [9], hydrophilicity [10], and antifouling [11] are just a few examples of what can be accomplished through surface modification. Surface modification can involve simple exposure of the membrane to a chemical or a physical treatment, such as plasma, in order to achieve the desired modification of properties. However, the durability of these simple treatments is normally found to be low. Over time, the improved properties from such treatments degrade, and are no longer effective. Therefore, many studies have been conducted on surface modification of membranes with grafting of another material, normally a monomer with reactive groups, on to the surface. The grafting of materials to the membrane surface through various procedures such as chemical, UV-initiated grafting, plasma grafting, and ozone grafting are normally quite stable, as the materials are covalently bonded to the membrane surface [12]. 


\section{1-4-1. UV-Initiated Grafting}

UV-initiated grafting involves exposing a membrane to ultraviolet wavelengths of light in order to initiate reactions between the membrane surface and grafting material. Ultraviolet light is defined as wavelengths of light that fall between $10 \mathrm{~nm}-$ $400 \mathrm{~nm}$. However, UV-initiated grafting for membrane surface modification is often conducted with wavelengths of light between $200 \mathrm{~nm}-400 \mathrm{~nm}[13,14]$. In order for UV-initiated grafting to occur, a photoinitiator is needed. A photoinitiator is a lightsensitive compound that interacts with UV light, normally through chain scission or hydrogen abstraction, in order to generate free radicals that can initiate reactions between membrane surfaces and chosen grafting materials [15]. The generated free radicals can initiate reactions which covalently link the chosen grafting material to the surface of a membrane, creating a durable surface modification. Benzophenone is a common aromatic ketone photoinitiator used in UV-initiated grafting. 


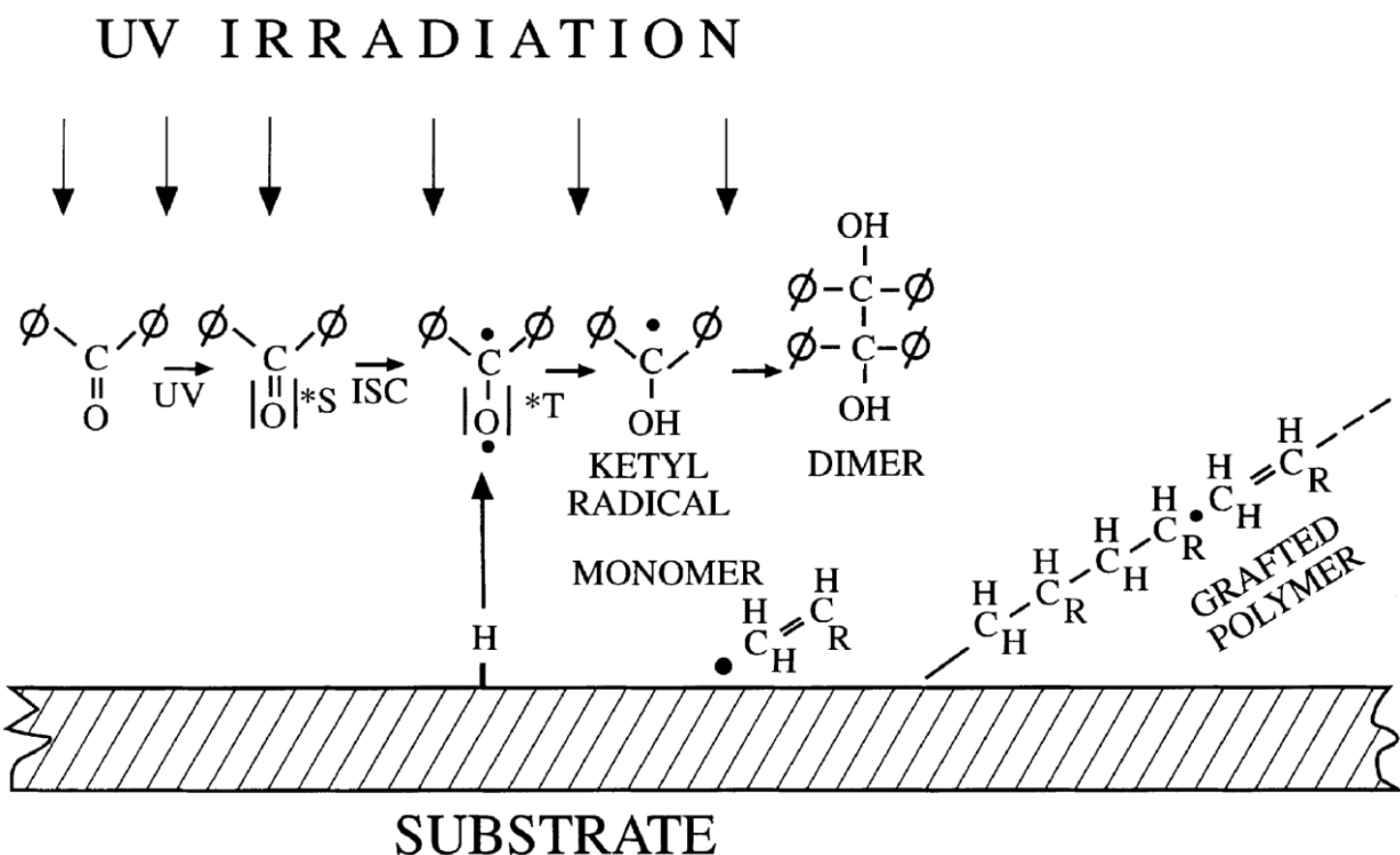

Figure 2. Mechanism of UV-initiated surface grafting with benzophenone as the photoinitiator and an inert substrate surface [16]

Figure 2 depicts the mechanism of radical generation by UV exposure to the photoinitiator and the subsequent hydrogen abstraction of an inert substrate surface, such as polypropylene. The radical generated on the photoinitiator, in this case benzophenone, is stabilized by two phenyl groups. This radical can then abstract a hydrogen from the substrate surface, generating a radical on to the substrate. This radical can then react with carbon-carbon double bonds of the chosen grafting material and create a covalent bond between the substrate surface and the grafted material. In the case of this current study, the radical generated by the photoinitiator can also initiate the cross-linking reactions between PEGDA chains, forming a hydrogel which will be grafted on to the surface of a microporous polypropylene membrane. 


\section{1-4-2. Plasma Grafting}

Plasma grafting for surface modification involves exposing the surface to plasma, often generated from high energy sources such as radio waves, laser, or microwaves under an inert gas. The plasma radiation creates free radicals on the surface of the material, which sometimes is then allowed to react with oxygen in order to form peroxides [17]. These peroxides are exchanged in solution with the chosen grafting material, often molecules containing carboxyl, hydroxyl, or amine groups. Some plasma grafting methods instead allow the grafting material to react with the plasma generated free radicals on the substrate surface before they can react with oxygen to form peroxides. This can be accomplished simply by exposing the surface, submerged in the grafting material solution, to plasma under an inert gas such as helium or argon. Due to the high energy of plasma radiation, damage to the substrate material is a common issue. One method for dealing with this is to minimize the power input by exposing the substrate surface to plasma in pulses.

\section{1-4-3. Ozone Grafting}

Ozone grafting also involves peroxides generated on the substrate surface. This is often accomplished by exposing a substrate to ozone gas, which can be generated from oxygen with the use of a high voltage source. The peroxides can then be decomposed by redox reaction and the grafting reaction with the chosen monomer can occur. Exposure to excessive ozone can also cause damage to the substrate material, decreasing the mechanical properties [10]. 
The main benefit of the three aforementioned grafting procedures is their ability to quickly and easily modify the surfaces of substrate materials which are normally chemically inert, such as polypropylene, a polymer which does not contain any functional groups. Chemically inert polymers can be functionalized using these grafting methods, or monomers can be directly grafted on to the surface. One major drawback to these grafting procedures is the possible damage to the substrate material with excessive exposure to the high energy sources. Another issue is the homopolymerization of the chosen grafting material, leading to a lower grafting yield on the substrate surface. Overall, these surface modification techniques are viable and useful for improving various properties of a substrate material.

\section{1-5. Grafting with Poly(ethylene glycol)}

In this current study, PEGDA was grafted to the surface of a microporous polypropylene membrane. The grafted PEGDA was shown to reduce the pore sizes of the membrane. When exposed to moisture, PEGDA is expected to swell significantly, reducing the pore sizes even further. A microporous membrane that has been grafted with PEG has shown similar properties. Tan and Obendorf [18] developed a moistureresponsive membrane for use as protective clothing. This membrane was created by graft polymerizing poly(ethylene glycol) (PEG) on to the surface of a microporous polyurethane (PU) membrane. This resulted in a PU membrane with a smaller pore size distribution which provides greater protection from pathogens. The membrane's pore sizes were further reduced when exposed to moisture due to the swelling of the hydrophilic PEG. However, the rate at which the grafted PEG swelled was quite low 
and may not reduce the pore size in time to prevent penetration from liquid-borne pathogens.

PEGDA, the acrylate functionalized version of PEG, is a hydrogel with good swelling properties. For this current study, PEGDA is expected to provide a greater degree of protection due to the increased swelling properties as well as the ability to form a cross-linked network [7]. The acrylate groups on both ends of PEGDA will allow for bonding between the PEGDA and polypropylene surface as well as between PEGDA macromolecules, allowing the degree of pore size decrease to be controlled based on the size of the cross-linked PEGDA network. In this current study, a UVinitiated grafting method was used instead of a chemical grafting method such as that chosen by Tan et al. [18]; the UV-initiated method is expected to be simpler and use less harmful chemicals. Polypropylene was chosen over polyurethane as the microporous membrane for this current study due to some of its advantages, such as chemical stability and cost.

\section{1-6. Grafting with Acrylates}

\section{1-6-1. N,N-dimethylamino ethyl methacrylate}

The two acrylate end groups on PEGDA provide alkene groups which allow for grafting of the macromolecules to the surface of a polypropylene membrane as well as to other PEGDA molecules through homopolymerization. This will allow for the amount of pore size reduction to be controlled by the degree of PEGDA grafted to

the membrane surface. The degree of grafting has been shown to be dependent on the concentration of the macromolecule absorbed on to the membrane surface before 
grafting as well as the grafting conditions. Shaofeng et al. [19] immobilized N,Ndimethylamino ethyl methacrylate (DMAEMA), in which only a single alkene group is available for grafting, on to the surface of a microporous polypropylene membrane using atmospheric-pressure plasma. This study showed the increase of hydrophilicity obtained by the surface modification through water contact angle analysis. The decrease in pore size of the membrane was observed simply though scanning electron microscopy (SEM). This study mainly demonstrated the significant effect of the plasma treatment conditions on the degree of immobilization of the monomer on to the membrane surface. Generally, increased plasma treatment time, increased discharge power, and increased frequency led to a greater amount of monomer immobilized, with a maximum at certain conditions.

The chosen monomer for their study, DMAEMA, possesses a single acrylate group which allows for bonding to the membrane surface by plasma grafting. However, homopolymerization occurred between DMAEMA monomers, leading to a decrease in the amount immobilized to the membrane surface. In this current study, PEGDA was chosen due to the presence of two acrylate end groups. Homopolymerization still occurs; however, the difunctionality of PEGDA is expected to improve grafting while allowing for a cross-linked PEGDA network to be bonded to the membrane surface. This current study also examines the effect of UV grafting conditions on the grafting degree of PEGDA to the surface of polypropylene membranes. 

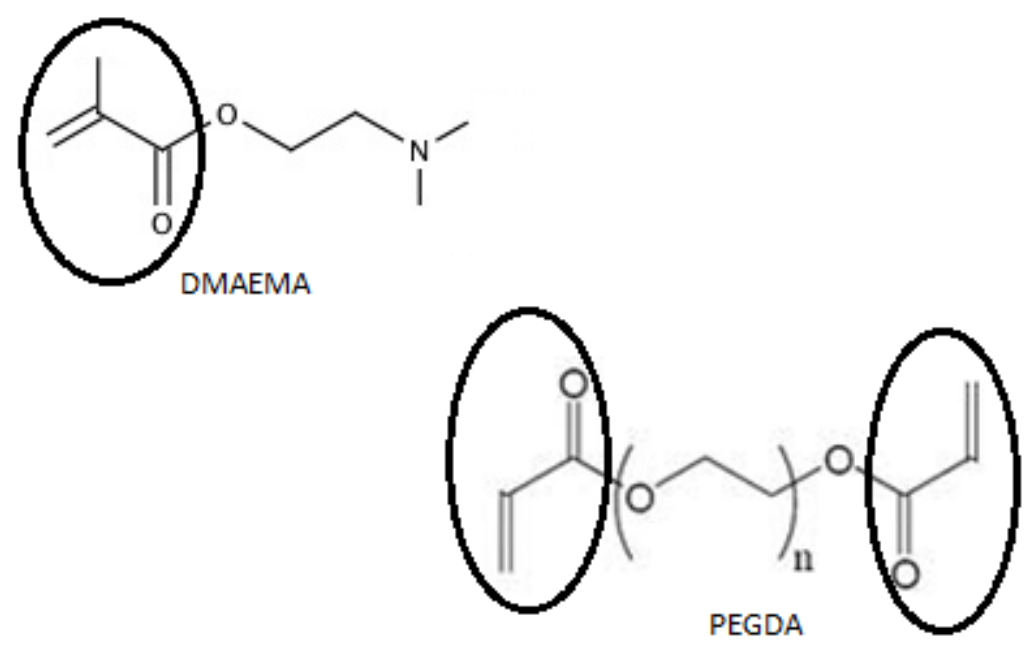

Figure 3. Comparison of the monofunctionality of DMAEMA to the difunctionality of PEGDA

Figure 3 shows the structures of DMAEMA and PEGDA. The DMAEMA possesses only one acrylate group available for grafting and reactions, while the PEGDA possesses two, providing difunctionality. While the acrylate group available on DMAEMA allows for grafting and homopolymerization reactions to occur, it does not have the ability to form a three dimensional cross-linked network, also known as a hydrogel, that PEGDA has due to its difunctionality.

\section{1-6-2. D-gluconamidoethyl methacrylate}

Another study involving acrylate grafting, conducted by Yang et al. [20], grafted a novel sugar-containing monomer, D-gluconamidoethyl methacrylate (GAMA), on to a microporous polypropylene membrane through UV-induced polymerization. This was accomplished by exposing microporous polypropylene membranes to a photo-initiator solution, benzophenone in heptane, and then briefly 
drying in air. The membranes were then washed with acetone and fixed between two filter papers. They were then immersed in varying concentrations of the GAMA monomer aqueous solutions. UV irradiation was then conducted on these solutions and membranes under an argon gas environment. The resulting membranes were then washed with water and dried.

The concentration of the monomer solution was shown to have a strong effect on the degree of grafting, or amount of monomer grafted to the membrane surface. However, as the monomer concentration increased past a certain level, the degree of grafting did not increase as significantly. This is due to homopolymerization of the monomer as well as limited active grafting sites on the membrane surface. Increased UV irradiation time was also shown to increase grafting degree, although a maximum level was observed as well. SEM images in this study show the decreasing pore sizes of the polypropylene membranes as grafting degree of the monomer increases. Contact angle measurements showed the increased hydrophilicity of the membrane after the surface treatment. This study demonstrates the feasibility of UV grafting of acrylate groups to the surface of polypropylene membranes. This current study has achieved similar results, but the chosen grafting polymer, PEGDA, has two available acrylates compared to the single acrylate group of GAMA. This difunctionality has provided slightly different and improved results. 


\section{1-6-3. Acrylic acid}

In one study conducted by Li et al. [21], another suitable grafting method was used for surface modification with acrylates: ozone induced grafting. This study grafted acrylic acid onto the surface of a microporous polypropylene membrane through ozone induced grafting in order to create a pH sensitive material. Ozone was generated with oxygen flow through an ozone generator in order to form active peroxides on the surface of the membrane to initiate the radical polymerization of the acrylic acid monomer. In this study, the membranes were first treated with ozone for a given period of time, and then soaked in ethanol. The membranes were then immersed in acrylic acid solutions and the grafting reaction was allowed to proceed under nitrogen.

This group found that with increased ozone exposure time and acrylic acid monomer concentration, the grafting degree would increase somewhat linearly. However, as the ozone exposure time increased, the mechanical properties of the membranes were found to decrease. As ozone exposure increased, the polypropylene membranes would begin to degrade and tensile properties were decreased. Li et al. also found that with increased grafting reaction temperature, the grafting degree would increase as well due to an increased amount of free radicals generated as well as increased diffusion of the acrylic acid monomer in to the membrane. However, a peak temperature was reached, and grafting degree began to decrease after a certain temperature due to the life span of free radicals decreasing from the higher temperatures. Water permeability tests showed a decrease in water permeability of the treated membranes as $\mathrm{pH}$ increased. This was due to the $\mathrm{pH}$ sensitivity of the 
poly(acrylic acid) (PAA) which was grafted to the surface of the membrane. As $\mathrm{pH}$ increased, the PAA uncoiled and decreased the pore sizes of the polypropylene membrane, thus decreasing water flux through the membrane.

Similar results were found in this current study, although UV-initiated grafting was the chosen grafting method. UV exposure conditions were also found to affect the grafting degree, where UV exposure time would increase linearly with grafting degree. However, the polymer concentration (PEGDA) was found to be related in an exponentially increasing manner with grafting degree, rather than the linear relationship of acrylic acid monomer concentration and grafting degree. This is due to the difunctionality of PEGDA compared to the single acrylate group available from the acrylic acid monomer. Responsiveness of this current study's membrane was targeted at moisture exposure, rather than $\mathrm{pH}$ sensitivity. Moisture exposure to the membrane causes swelling of the grafted PEGDA and subsequent decrease in pore sizes.

\section{1-7. Grafting with Acrylated Poly(ethylene glycol)}

\section{1-7-1. Plasma Grafting}

PEGDA is expected to possess superior swelling properties to PEG due to its ability to form a cross-linked network. This cross-linking ability is provided through the two acrylate end groups which have replaced the hydroxyl end groups of PEG. The two acrylate ends provide alkene groups which can form covalent carbon-carbon bonds, through grafting and subsequent cross-linking, between a membrane surface and other PEGDA chains. This ability has been shown to improve the grafting of 
PEGDA versus PEG to a membrane surface. Iwata et al. [22] plasma grafted PEGDA on to a polysulfone membrane in order to impart anti-oil staining properties. They compared the oil penetration of both PEG and PEGDA grafted membranes. PEGDA grafted membranes sustained anti-oil properties for a greater amount of time than the PEG grafted membranes. This was likely due to the improved grafting achieved by the available acrylate groups of PEGDA. Their study also provided a very good schematic, shown in Figure 4, on the plasma grafting mechanism of PEGDA to a membrane surface.

(a)

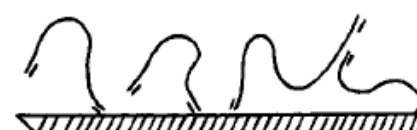

(b)
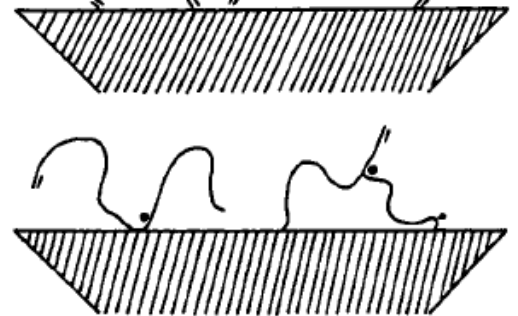
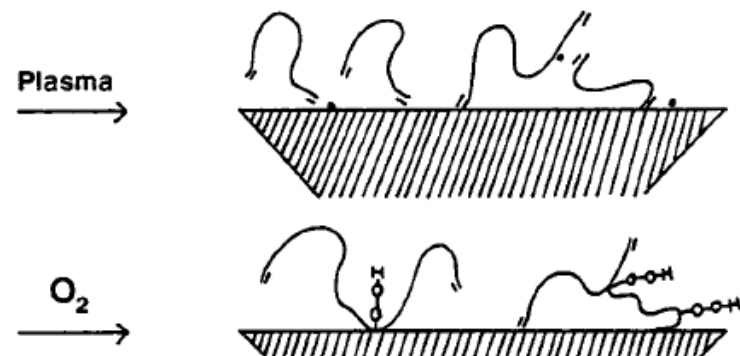

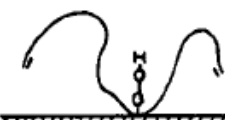

एuI

Figure 4. Plasma grafting of PEGDA on to membrane surface (a) radicals formed from plasma exposure through chain scission (b) bonds form between radicals and acrylate end groups, exposure to oxygen neutralizes radicals in to peroxides [22]

Radicals are generated on the membrane surface as well as on PEGDA chains by the plasma, causing bonds to form between the radicals and acrylate groups. The radicals then form peroxides when exposed to oxygen, stopping the grafting reactions. For this current study, varying concentrations of PEGDA was used in order to observe, by weight measurements and microscopy, the amount immobilized on the membrane surface, and the subsequent decrease in pore size. The swelling properties, rather than oleophobic properties, of PEGDA are focused on in this current study. 


\section{1-7-2. Ozone Grafting}

A study conducted by Ko et al. [23] compared the ozone grafting of monoacrylated PEG (PEGMA) and sulfonated monoacrylated PEG (PEGMA-SO ${ }_{3}$ ) to various polymer films such as polymethylmethacrylate (PMMA), polyethylene (PE), polyurethane (PU), and silicone. The ozone grafting was accomplished by exposing the various films to ozone under oxygen in order to form peroxides on the surface. After ozonation, the films were immersed in aqueous solutions of the PEG derivatives and kept under nitrogen in order for the grafting reaction to occur between the peroxides on the surface of the films and the PEG derivatives' end groups. The treated films were then washed to remove unreacted PEG.

This study showed that PU and silicone exhibited the highest peroxide concentrations of the films after ozone exposure. The authors speculate that this is due to the soft structures of PU and silicone having high ozone permeability. PE, on the other hand, had a lower peroxide concentration due to the hard and crystalline structure which does not allow as much ozone to penetrate the polymer. The study conducted by Ko et al. [23] also showed a decrease in water contact angle of the films after the ozone exposure step. This shows that the presence of peroxides on the surface is enough to increase the hydrophilicity of the films. However, after grafting of the PEG derivates to the surface, the contact angles decreased more significantly due to the hydrophilicity of PEG. The PEGMA grafted films exhibited lower contact angles than the $\mathrm{PEGMA}-\mathrm{SO}_{3}$ grafted films even though $\mathrm{PEGMA}-\mathrm{SO}_{3}$ contains strong hydrophilic ionic groups. The authors believe this is due to a low grafting yield of PEGMA-SO $\mathrm{S}_{3}$ in comparison to PEGMA. This could indicate that ionic groups may 
disrupt the grafting reaction, and for this current study, PEGDA may be a more suitable grafting material than a sulfonated derivative.

\section{1-7-3. UV-Initiated Grafting for Responsive Macropores}

PEGDA macromolecules grafted to the membrane surface have been shown to reduce the pore sizes physically. The swelling properties of PEGDA are expected to provide moisture-responsiveness to the pores. When exposed to moisture, the PEGDA grafted to the membrane surface, which partially fills the pores, is expected to swell and further reduce the pore sizes. This concept has been successfully shown on a macro-scale. In unpublished work by Fei Song and Jin-tu Fan of The Hong Kong Polytechnic University, a moisture sensitive pore was created in a polyurethane membrane using a complex of PEGDA and $\alpha$-cyclodextrin with UV photo-crosslinking. A pore was cut in to a polyurethane membrane using plasma etching. This pore was then filled with a PEGDA and $\alpha$-cyclodextrin complex solution. This solution was then cross-linked with UV irradiation. The resulting pore was slightly decreased in size. When exposed to moisture, the PEGDA complex swelled significantly and decreased the pore size further. When dried, the PEGDA contracted, causing the pore to return to almost its original size. For this current study, the microporous structure of the whole membrane was modified, rather than a single

artificially created pore. The PEGDA swelling from exposure to moisture is expected to show similar pore size reducing properties. 


\section{1-7-4. UV-Initiated Grafting on Poly(dimethylsiloxane)}

There have been many studies done on poly(dimethylsiloxane) (PDMS) photoinduced surface grafting with PEGDA. In one such study, done by Sugiura et al. [24], both poly(ethylene glycol) monoacrylate (PEGMA) and PEGDA were surface photografted with UV light on to PDMS. This was accomplished by immersing a PDMS plate in a benzophenone, their chosen photo-initiator, solution in acetone. The acetone was then washed away with water and the PDMS surface was exposed to an aqueous PEGMA or PEGDA solution. This was covered by a photomask and then exposed to UV light. The result was a PDMS surface with increased hydrophilicity and decreased water contact angle.

The purpose of this study was to show the decreased protein adsorption and cell adhesion to the PEG grafted areas. However, this study also showed that with the PEGMA graft, the increased hydrophilicity of PDMS was gradually lost within a period of two weeks. This could mean that the PEGMA graft to the PDMS surface was not very stable. On the other hand, the PEGDA graft exhibited a stable increased hydrophilicity for over two months. The data from this study also suggest that with an increased degree of cross-linking, through increased UV exposure time, the stability of the PEGDA graft to the PDMS surface increases. The authors suggest that the stable hydrophilicity of the PEGDA graft could be due to the benzophenone diffusing in to the PEGDA, initiating cross-linking. This cross-linked PEGDA then strongly grafts to the PDMS surface. This could indicate that the cross-linking of PEGDA could play a larger role in the grafting to the PDMS surface than simply the chemical interactions between the end groups of PDMS and the PEGs. 
This current study has also successfully photo-grafted PEGDA on to a surface and shown stable increased hydrophilicity. Benzophenone was also the chosen photoinitiator and PEGDA is expected to form a cross-linked network which grafts to the surface of a microporous polypropylene membrane. However, more focus was on the porous structure of the substrate and the effects of grafting on pore sizes rather than protein adsorption and cell adhesion.

In this current study, moisture-responsive pores were achieved through the swelling of PEGDA in response to moisture. The amount of swelling which occurs in PEGDA depends on its molecular weight [25]. It has been shown that a PEGDA hydrogel formed from lower molecular weight polymers will form a compact structure with a higher modulus. Thus, the lower molecular weight hydrogel will be stiffer and expand less when exposed to moisture. Generally, higher molecular weights of PEGDA will form less compact cross-linked networks, allowing for a greater amount of water to penetrate and swell the structure. Three molecular weights of PEGDA were used in this current study: 258, 575, and 700 . However, more focus was on PEGDA 575 due to ease of handling.

\section{1-8. Objectives}

The goal of this study is to modify a hydrophobic microporous polypropylene membrane through UV-initiated grafting with PEGDA. This modification will improve hydrophilicity of the membrane, modify the pore sizes, and create moistureresponsive pores; namely, pores which further reduce in size when exposed to 
moisture. This membrane will provide protection without a complete sacrifice of comfort, as water vapor will still be transported through the pores. When exposed to moisture, or possible liquid-borne pathogens, the membrane pore sizes will decrease further and provide a greater degree of protection.

The grafting procedure is expected to form covalent bonds between the PEGDA chains and the membrane surface. PEGDA chains are also expected to covalently bond to each other, forming a three dimensional cross-linked network, also known as a hydrogel, which is attached to the surface of the membrane. This is expected to provide a durable surface modification of the microporous polypropylene membrane. This membrane should retain its improved properties over a long period of time, and various solvents should be unable to remove the grafted PEGDA. 


\section{CHAPTER 2}

\section{EXPERIMENTAL}

\section{2-1. Materials}

Microporous polypropylene membranes were acquired from Sterlitech Corporation, Kent, WA. The membranes were disc shaped with a diameter of $47 \mathrm{~mm}$, average pore size of $0.2 \mu \mathrm{m}$, and thickness of about $150 \mu \mathrm{m}$. Poly(ethylene glycol) diacrylate $\left(M_{n} 258,575\right.$, and 700) and benzophenone were purchased from SigmaAldrich Corporation, St. Louis, MO. Acetone and isopropyl alcohol were acquired from Mallinckrodt Chemicals, St. Louis, MO.

\section{2-2. Membrane Surface Modification}

\section{2-2-1. Procedure}

Microporous polypropylene membranes were soaked in about $10 \mathrm{~mL}$ of photo-initiator solution (10\% wt. benzophenone in acetone) for $20 \mathrm{~min}$. The membranes were then rinsed in water to remove excess benzophenone and allowed to air dry for $5 \mathrm{~min}$. These membranes were then immersed in 10 mL of PEGDA solutions consisting of various PEGDA weight percents in $75 \% \mathrm{H}_{2} \mathrm{O} / 25 \%$ isopropyl alcohol (w/w). A $\mathrm{H}_{2} \mathrm{O}$ / isopropyl alcohol (IPA) mixture was chosen as the PEGDA solvent in order to allow the microporous polypropylene membranes to wet out, since the high hydrophobicity of nascent polypropylene membranes would prevent PEGDA from penetrating the membrane when in purely aqueous solution. The membranes immersed in solution were then exposed to UV light in a UV chamber (Rayonet 
Model RMR-600, Southern New England Ultraviolet Company, Branford, CT) for 15 min. The effect of amount of UV exposure time as well as PEGDA molecular weight on grafting degree was examined. The treated membranes were then washed in acetone to remove unreacted benzophenone, sonicated in water to remove homopolymerized and unreacted PEGDA, and dried in a vacuum oven at ambient temperature. All samples were created in triplicate.

Membranes were weighed before and after surface modification, and the following equation was used to calculate the degree of grafting, or weight add-on of $\operatorname{PEGDA}\left(\mathrm{D}_{\mathrm{g}}\right)$ :

$$
D_{g}(\%)=\frac{W_{g}-W_{o}}{W_{o}} \times 100
$$

where $W_{g}$ is the weight of the PEGDA grafted membrane and $W_{o}$ is the weight of the nascent membrane. Any conditioning of the samples before characterization was done by storing samples overnight in a conditioning room maintained at a temperature of 21 ${ }^{\circ} \mathrm{C}$ with a relative humidity of $65 \%$. In order to condition at a theoretical $100 \%$ relative humidity, a sample was stored over deionized water in a sealed container within the aforementioned conditioning room. 


\section{2-2-2. Proposed Grafting Scheme}
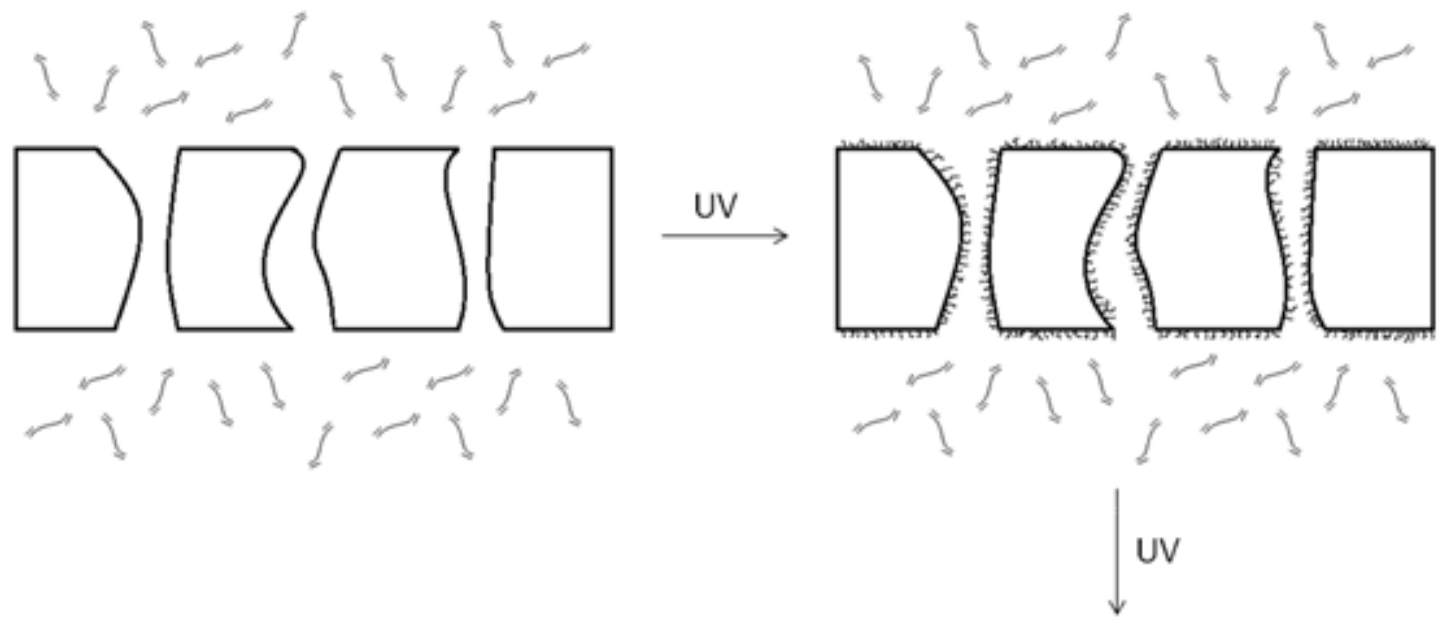

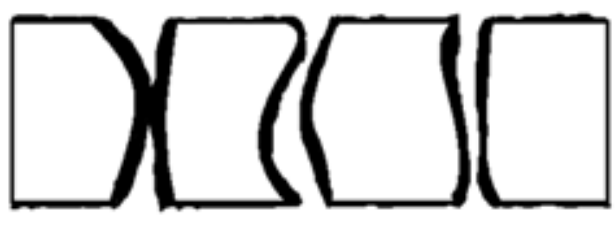

Swollen state $\underset{\text {-Moisture }}{\stackrel{\text { Moisture }}{\longleftrightarrow}}$

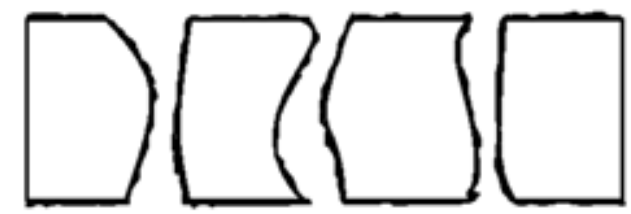

Moisture responsive membrane

Figure 5. Illustration of proposed schematic for the surface grafting of microporous polypropylene membrane's porous structure with PEGDA (a) membrane in PEGDA solution (b) initial graft of PEGDA chains to surface (c) further grafting to achieve moisture-sensitive membrane in non-swollen state (d) moisture swollen state

Figure 5 depicts an illustration of the proposed schematic for the UV-initiated surface grafting of microporous polypropylene membrane with PEGDA. (a) depicts the porous structure of the microporous polypropylene membrane substrate, where the surface pores are generally larger than the bulk, or through, pores. This membrane is surrounded in solution by PEGDA chains with the carbon-carbon double bonds, available through the acrylate end groups, depicted on each end of the chains. (b) shows the penetration of PEGDA chains into the porous structure of the membrane and the initial grafting of the chains to the surface with UV irradiation and photoinitiator. Penetration of the graft polymer into the substrate has been shown to be 
highly dependent on the solvent [26]. In this current study, a 75\% $\mathrm{H}_{2} \mathrm{O}$ / 25\% IPA solvent system was used and is believed to allow adequate penetration of PEGDA in to the porous structure of the substrate membrane in order for the surface grafting to modify the pore sizes.

Once the initial grafting of the PEGDA chains to the surface of the membrane has occurred, there are still acrylate groups available on the end of these chains, not depicted in the illustration, that allow for the cross-linking of PEGDA to occur under further UV irradiation with photoinitiator. (c) depicts further grafting of PEGDA onto the substrate surface after further UV exposure with photoinitiator. The un-reacted PEGDA chains still available in solution can cross-link and bond with the available acrylate group of the PEGDA already grafted to the surface of the membrane. This will create a thin layer of PEGDA hydrogel grafted to the surface of the microporous polypropylene membrane. This thin layer of PEGDA, dried so that it is not swollen with water molecules, will cause the pore sizes of the membrane to decrease depending on the amount of PEGDA grafted. (d) shows the swollen state of the PEGDA graft when exposed to moisture. When exposed to moisture, through change in relative humidity or a liquid-borne pathogen, the PEGDA hydrogel grafted to the substrate surface will absorb the water molecules and swell in response. This swelling will cause a further decrease in the pore sizes of the grafted membrane, enhancing the degree of protection. Once moisture is removed from the system, the grafted PEGDA will contract back to its original non-swollen state as depicted in (c). 


\section{2-3. Characterization}

\section{2-3-1. Grafting Conditions}

The grafting conditions effects on grafting degree $\left(D_{g}\right)$ were examined by plotting the grafting degree as the dependent variable and the various grafting conditions as the independent variables. All grafting degrees were measured after samples were vacuum dried overnight in an attempt to remove all water content. The grafting conditions examined are the PEGDA grafting solution concentration (wt. \%), the molecular weight $\left(\mathrm{M}_{\mathrm{n}}\right)$ of the PEGDA, and the amount of UV exposure time (minutes). All plots in this study were created with SigmaPlot 9 (Systat Software Inc., San Jose, CA).

\section{2-3-2. Surface Morphology}

The surfaces of the microporous polypropylene membranes were examined by scanned electron microscopy (SEM, Leica Stereoscan 440, Leica Microsystems GmbH, Wetzlar, Germany). Samples were sputter coated with Au-Pd for 30 s before SEM analysis. SEM analysis was used simply to visually assess the PEGDA graft to the surface of the microporous polypropylene membranes with varying grafting degrees.

\section{2-3-3. Fourier Transform Infrared Spectroscopy}

In order to confirm successful grafting of PEGDA to the surface of microporous polypropylene membranes Attenuated Total Reflectance Fourier Transform Infrared Spectroscopy (ATR-FTIR, Magna-IR 560 Spectrometer, Nicolet 
Instrument Corporation, Madison, WI) was used. FTIR scans were done at $4 \mathrm{~cm}^{-1}$ resolution with 64 scans performed on each sample. Presence of PEGDA on the substrate was indicated by the identifying peaks located on the acrylate end groups [25]. Figure 6 below shows the FTIR spectra of PEG compared with PEGDA. The peak at $1630 \mathrm{~cm}^{-1}$ corresponds to the carbon-carbon double bonds of the acrylate end groups while the peak at $1730 \mathrm{~cm}^{-1}$ corresponds to the carbonyl groups.

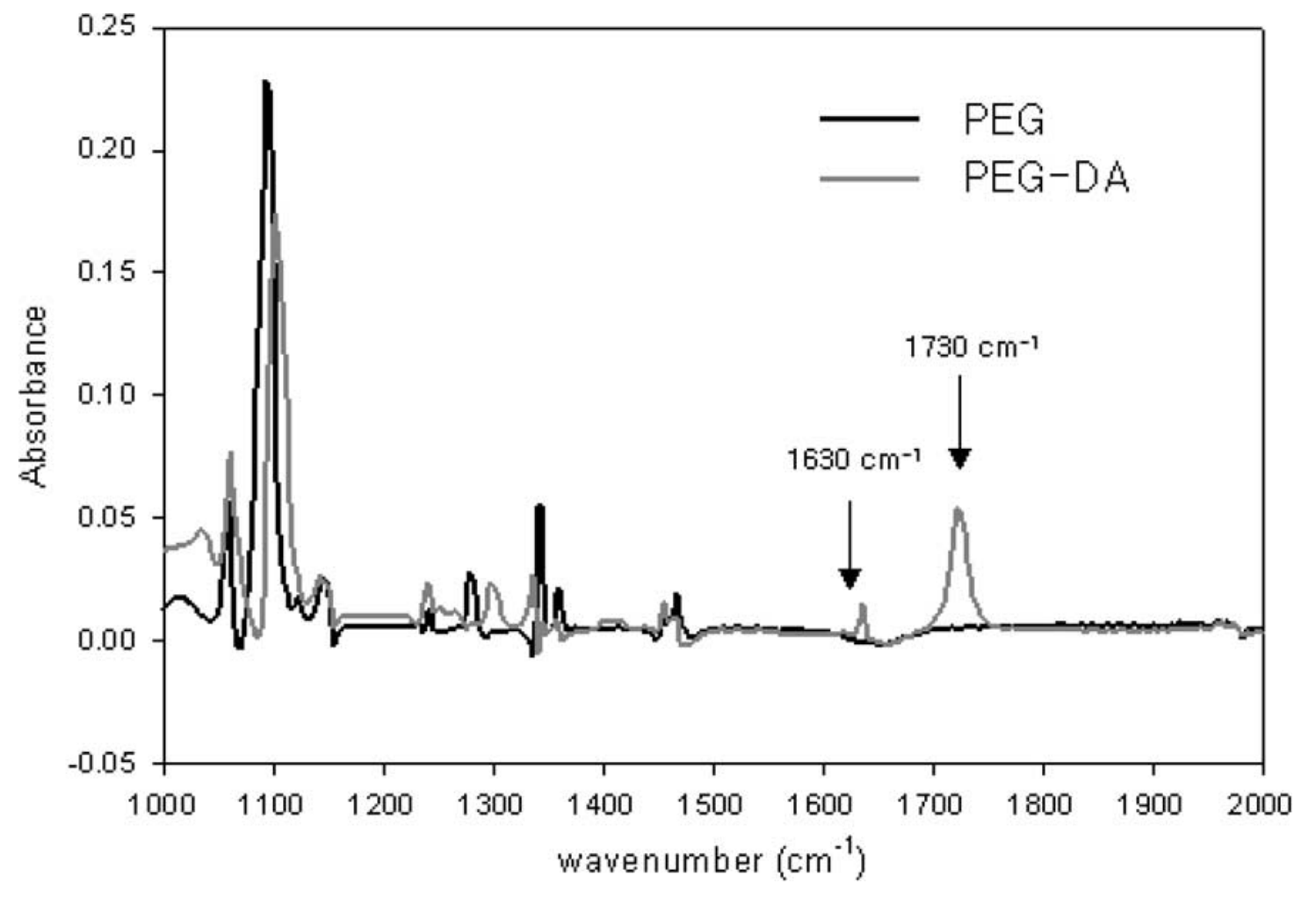

Figure 6. FT-IR spectra of PEG and PEGDA [25] 


\section{2-3-4. Contact Angle Measurements}

The contact angles of water droplets on the surface of the membranes were measured using a Contact Angle Analyzer (Model CAA2, IMASS Inc., Accord, MA). All samples were conditioned overnight at $21^{\circ} \mathrm{C}$ and at a $65 \%$ relative humidity before measurements were taken. Droplets of deionized water were delivered on to the surface of samples through a syringe and needle. The droplets' height and width were recorded and used to calculate the contact angles. Measurements were taken from at least three random locations on each sample. Contact angles were calculated with the following equation:

$$
\cos \theta=\frac{\left(x^{2}-y^{2}\right)}{\left(x^{2}+y^{2}\right)}
$$

where $x$ is the $1 / 2$ droplet width and $y$ is the droplet height. $\theta$ is the contact angle. Higher contact angle measurements indicate greater hydrophobicity of the sample. In this study, nascent microporous polypropylene membranes are relatively hydrophobic, while the grafting material, PEGDA, is highly hydrophilic. Contact angle measurements show the change in hydrophilicity of the grafted samples.

\section{2-3-5. Porometry}

Pore size analysis on the membranes was conducted with a Capillary Flow Porometer (Model CFP-1100-AEHXL, Porous Materials Inc., Ithaca, NY). This method involves forcing air with incrementally increasing pressure through the material and measuring the amount of air flow allowed to pass through. The pressures and the corresponding air flows allowed through the samples are plotted. Generally, 
smaller pore sizes allow less air flow to pass through. The sample is then wetted with a liquid of known surface tension (20.1 dynes/cm). This liquid blocks all the pores of the samples, preventing any air flow through the sample until a critical pressure is reached. Once the critical pressure is reached, the wetting liquid will be forced out of the largest pores first. As pressure continues to increase, the smaller pores will empty as well, allowing air flow to once again pass through.

These pressures and their corresponding air flows are also plotted. The plot of the dry air flow sample is halved and is also included on the plot. With this information, a great deal of analysis can be done on the porous structure of the sample. Bubble point, mean flow pore diameter, and pore size distribution are some of the important measurements that can be made with further calculation. This is described in further detail by Jena and Gupta [27]. An example of the output from a capillary flow porometer can be seen in Figure 7 below. The bubble point occurs at the pressure where the wet curve begins to allow air flow. This corresponds to the wetting liquid being forced out of the largest pore. The mean flow pore diameter occurs where the wet curve intersects the half dry curve. 


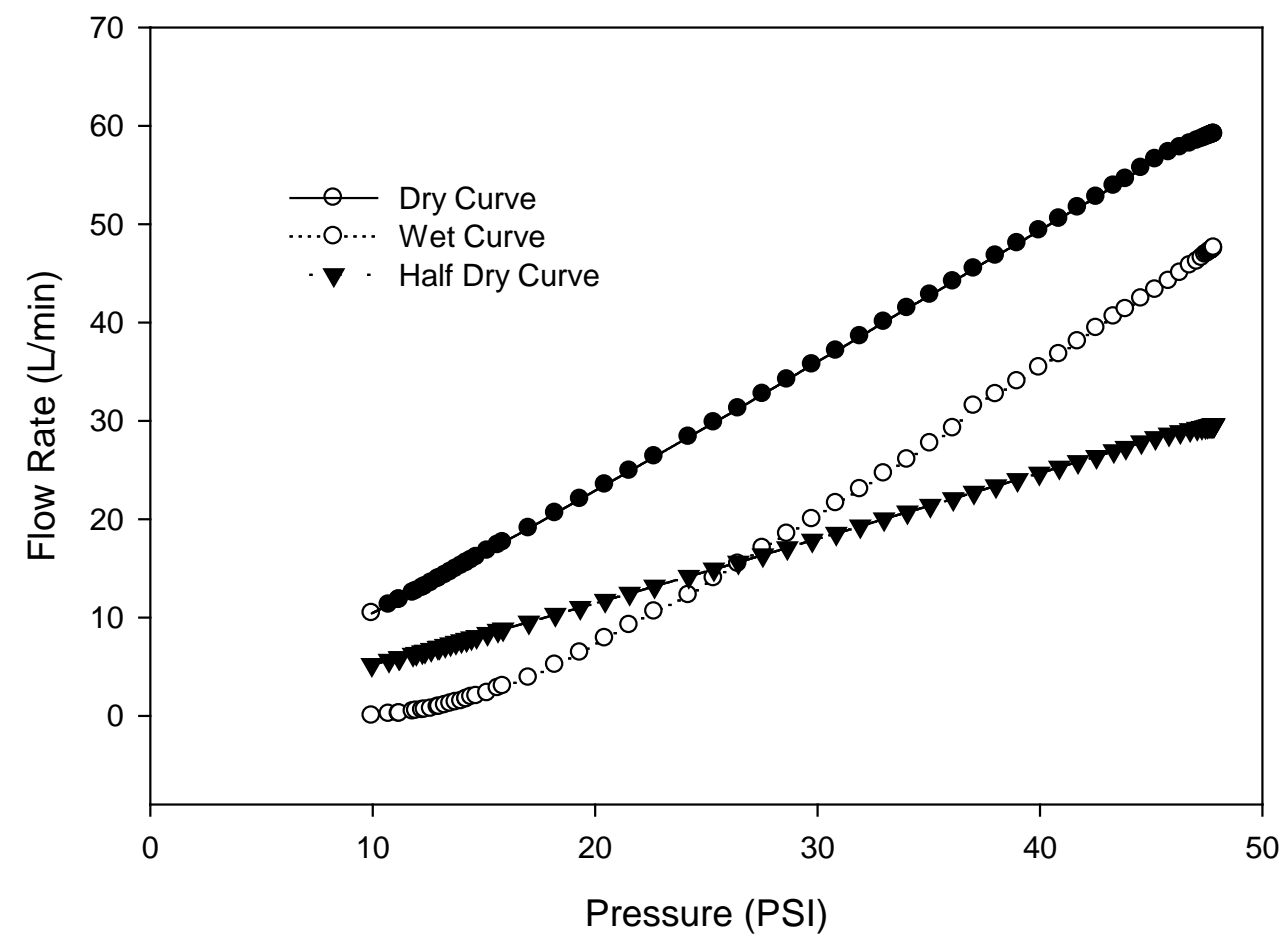

Figure 7. Capillary flow porometer output plot of an untreated microporous polypropylene membrane

\section{2-3-6. Statistical Analysis}

Analysis of covariance (ANCOVA) was conducted on the linear regressions of the porometry dry air flow data using JMP 9 (SAS Institute Inc., Cary, NC). ANCOVA was used to determine if there was a significant difference in slopes of the linear regressions. This can indicate whether the difference in grafting degree or relative humidity had a statistically significant effect on the amount of air flow measured passing through the sample. 


\section{CHAPTER 3}

\section{RESULTS AND DISCUSSION}

\section{3-1. Grafting Conditions}

\section{3-1-1. Solution Concentration}

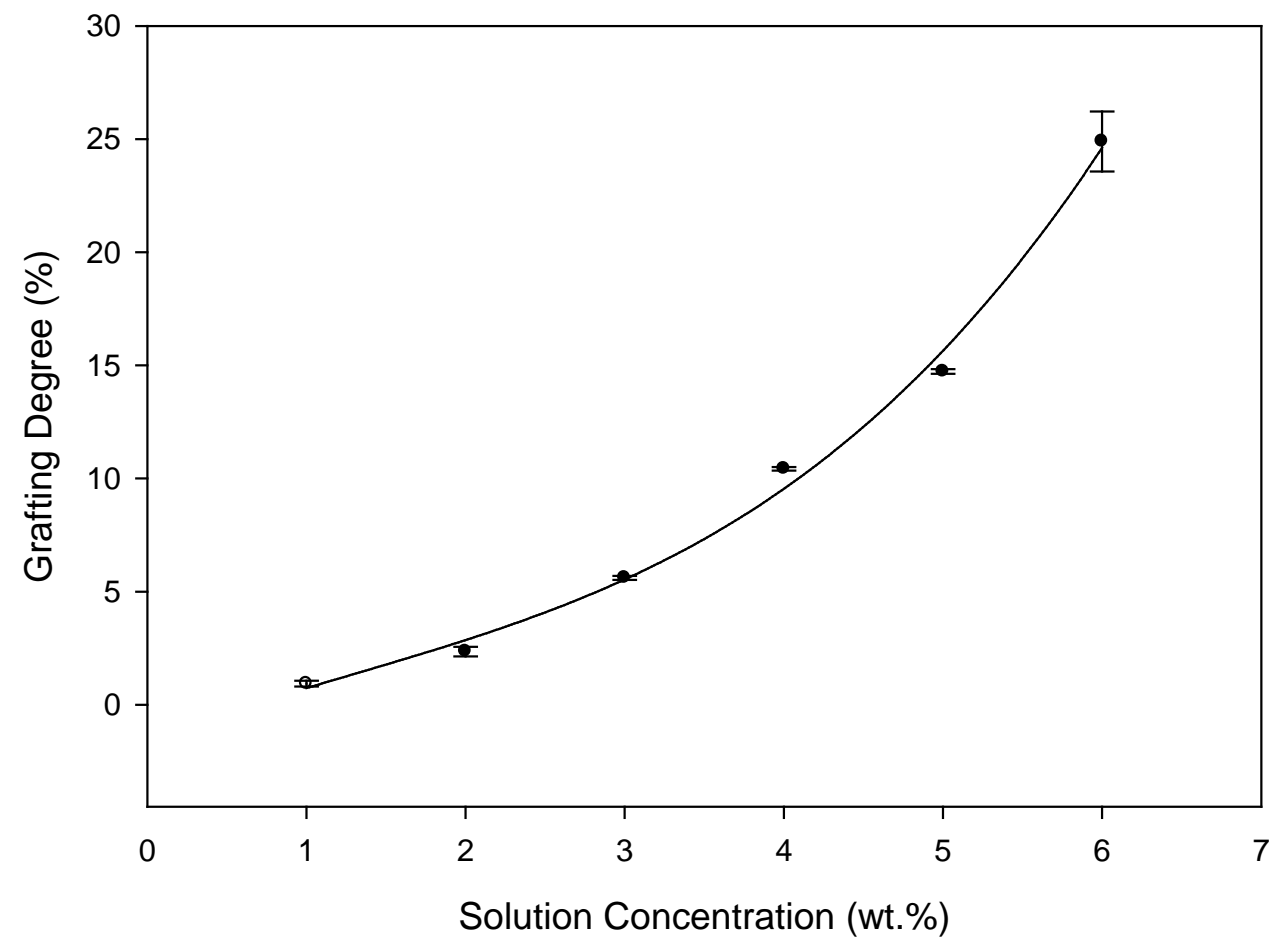

Figure 8. The effect of PEGDA grafting solution concentration on grafting degree with PEGDA $M_{n} 575$ and 15 min of UV exposure

Figure 8 shows the relationship between the PEGDA grafting solution

concentration condition with the grafting degree of the treated membrane. The grafting degree, which is essentially the weight add-on of PEGDA to the microporous polypropylene membrane, is highly dependent on the grafting conditions. These measurements were taken while holding the molecular weight of PEGDA constant at 575 and the amount of UV exposure constant at 15 minutes. For this particular 
condition, the grafting solution concentration is shown to have an exponential relationship with the grafting degree. The trend line shown on the plot can be described with the following equation: $y=0.1287 x^{3}-0.4954 x^{2}+2.7101 x-1.6067$ with an $\mathrm{R}^{2}$ value of 0.992 . The majority of measurements shown have very small error bars. However, the final measurement at a solution concentration of $6 \%$ has a large standard deviation. This was due to the high grafting degree achieved. Essentially the weight add-on of the grafted PEGDA became too great for the grafting procedure used, and the treated membrane sank to the bottom of the grafting vessel, a small Petri dish containing the membrane immersed in the grafting solution. This caused some grafting material to be lost to the sides of the grafting vessel, resulting in a large standard deviation of the sample.

In the previously described literature where a monofunctional grafting material was chosen [19, 20], the plots of a similar comparison between the grafting solution concentration and grafting degree shows an exponential relationship, but with a decreasing slope. In the case of this current study, the relationship is shown to have an increasing slope. This is likely due to the difunctionality of the chosen grafting material, PEGDA. One end of a PEGDA chain can be grafted to the surface of the membrane, consuming a carbon-carbon double bond of one acrylate group while leaving the other end of the PEGDA chain available for possible cross-linking reactions. This is essentially the aim of this current study: to graft a relatively thin layer of PEGDA hydrogel to the surface of a microporous polypropylene membrane. The results of this measurement showing the effect of grafting solution concentration on grafting degree shows that the amount of PEGDA grafted to the surface of the 
membrane can be controlled to a large degree by controlling the grafting solution concentration. A small amount of PEGDA can be grafted to the membrane surface, or a theoretically infinite amount can; to the point where there is more hydrogel than the original substrate (over 100\% grafting degree).

Although not shown in these data, this study has achieved over $100 \%$ grafting degree. However, interestingly enough, the samples were damaged after drying, due to the removal of water from the grafted PEGDA hydrogel and the subsequent contracting of the cross-linked network. The damage was visible by the removal of color; the nascent microporous polypropylene membranes are white, but after grafting and drying of a grafting degree over $100 \%$, there was a large amount of color missing from the center of the membrane, replaced by the colorless hydrogel. This damage was not observed for any of the lower grafting degrees. 


\section{3-1-2. UV Exposure Time}

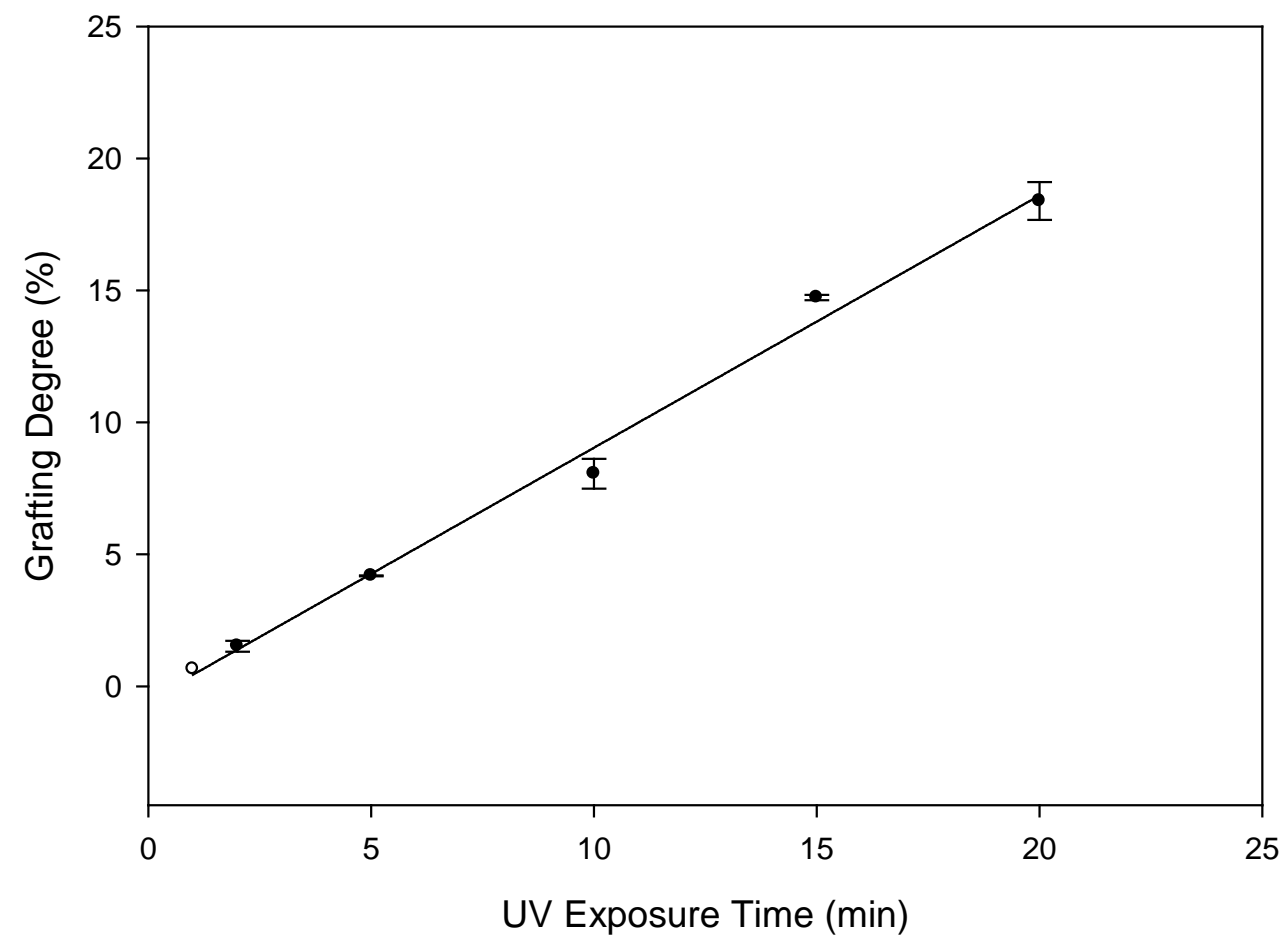

Figure 9. The effect of UV exposure time on grafting degree with PEGDA $M_{n}$ 575 and 5 wt.\% grafting solution concentration

Figure 9 shows the relationship between the amount of UV exposure, measured in minutes, and the grating degree of PEGDA on to the microporous polypropylene membrane. These measurements were made while keeping the molecular weight of PEGDA constant at 575 and the grafting solution concentration constant at 5 wt.\%. A fairly linear relationship with zero order kinetics is shown, and the trend line can be described by the following equation: $y=0.9555 x-0.5188$ with an $\mathrm{R}^{2}$ value of 0.9906 . The positive linear relationship is as to be expected. As the grafting solution is exposed to UV light for a longer period of time, more radicals will be generated, initiating more grafting and cross-linking reactions. However, not depicted in this plot, is the maximum grafting degree which is expected to be reached. 
Since the grafting solution concentration was held constant at $5 \mathrm{wt} . \%$, there is a limited number of PEGDA chains available for reactions. So unlike the previous comparison between grafting solution concentration and grafting degree, a theoretically infinite grafting degree is not possible for this condition. For this particular comparison between the condition of UV exposure time and grafting degree, the slope is expected to gradually decrease until a maximum grafting degree is reached; indicating that all the available PEGDA grafting material has been consumed in one of the following reactions: grafting to the surface of the substrate, cross-linking to these surface grafting chains, cross-linking with other PEGDA chains in solution (homopolymerization), or grafting to the surface of the grafting vessel. Obviously, the latter two are not included in the grafting degree calculation, as they are washed away from the grafted membranes before measurements are taken.

Previously published literature [28] has shown that polypropylene undergoes photo-oxidation when exposed to high energy light such as UV irradiation. This causes the polypropylene to have a decrease in physical properties such as the tensile properties. Essentially, greater exposure to UV light causes damage to the polypropylene. This current study did not study the effects of the UV-initiated grafting procedure on the mechanical properties of the substrate. However, with the relatively low UV exposure time, less than 20 minutes, significant damage to the microporous polypropylene membrane is not expected to occur, as previously published literature [28] has shown a polypropylene film maintaining its physical properties even after 21 days of UV exposure. 


\section{3-1-3. Molecular Weight}

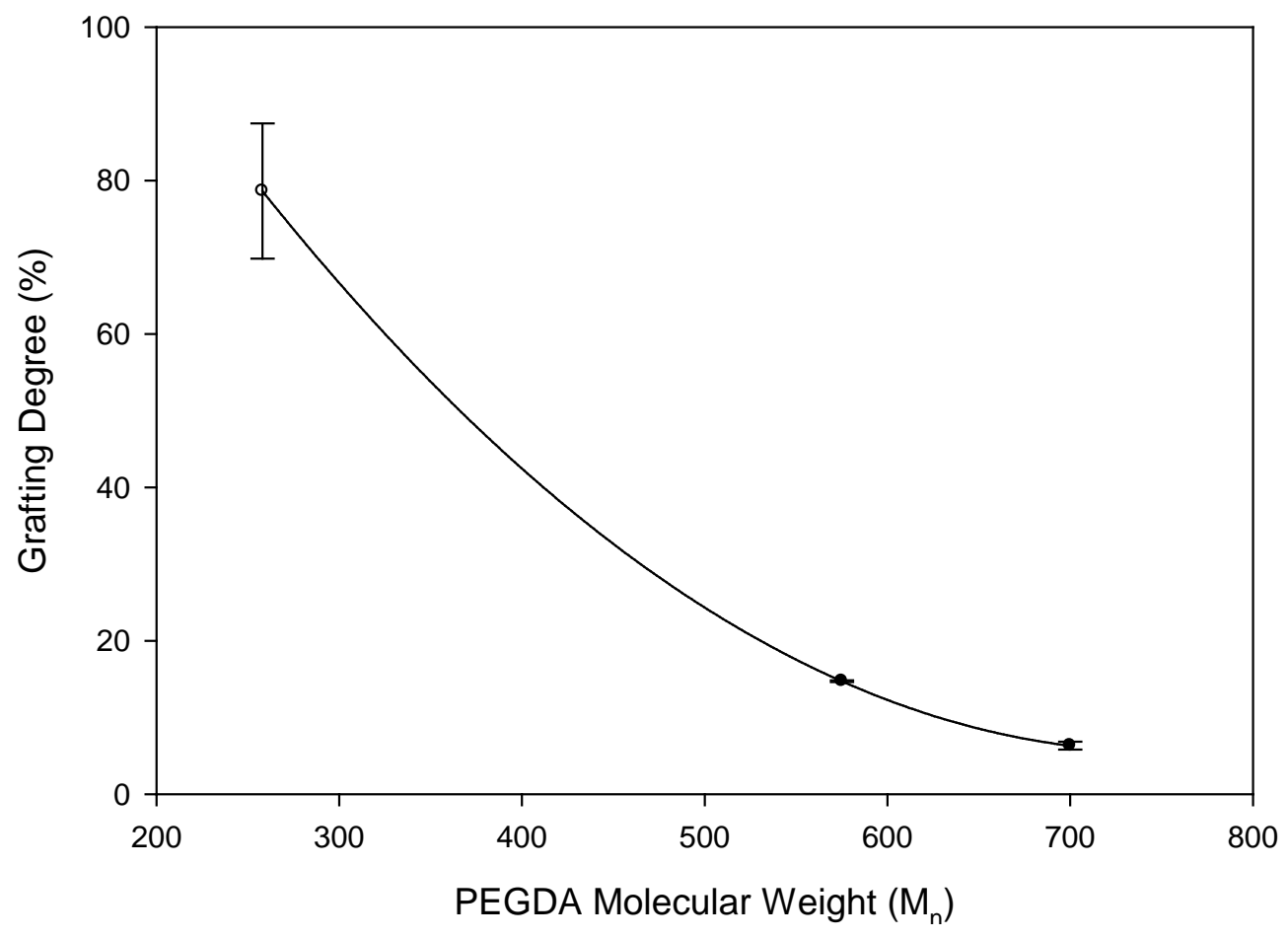

Figure 10. The effect of PEGDA molecular weight on grafting degree with 5 wt.\% grafting solution concentration and 15 min of UV exposure

Figure 10 shows the relationship between the PEGDA molecular weight and the grafting degree of the polymer to the substrate surface. These measurements were made while keeping the grafting solution concentration constant at $5 \mathrm{wt} . \%$ and the amount of UV exposure constant at 15 minutes. An exponential relationship can be seen and the trend line shown can be described by the following equation: $\mathrm{y}=$ $0.0003 x^{2}-0.4546 x+175.6918$ with an $R^{2}$ value of 0.9836 . The negative relationship between molecular weight and grafting degree is likely due to the fact that with higher molecular weights of the PEGDA polymer, the chains are longer. With longer polymer chains, the ends are less likely to find each other for cross-linking reactions, or even grafting reactions with the substrate surface. Generally, higher molecular weights of 
the polymer make the grafting reaction more difficult, therefore the grafting degree is lower. Once again, as similar to the grafting solution concentration versus grafting degree plot, a large standard deviation can be observed for the high grafting degree point, and relatively small error bars are shown for the lower grafting degrees. This is again due to the limitations of the chosen grafting vessel and solution. Once the grafting degree becomes too high, grafting material is lost to the sides of the grafting vessel. It is interesting to note that with lower molecular weights of PEGDA, extremely high grafting degrees are easily achievable, although homopolymerization occurs to a greater extent as well.

Although not examined in detail for this current study, the molecular weight of PEGDA not only has a strong effect on the grafting degree to the substrate surface, but it also greatly affects the degree of swelling of the resulting hydrogel. Generally, higher molecular weights of PEGDA result in larger cross-linked structures which can absorb a higher amount of water content [25]. This ability to absorb a higher amount of water content results in a greater degree of swelling. In the case of this current study, this would cause the pore sizes of the PEGDA grafted microporous polypropylene membrane to decrease to an even larger extent when exposed to moisture with higher PEGDA molecular weights. Although no detailed measurements have been made for this particular swelling property in this current study, it is believed that the molecular weight of PEGDA condition is an important factor for tailoring a moisture-responsive membrane. 


\section{3-2. Surface Morphology}
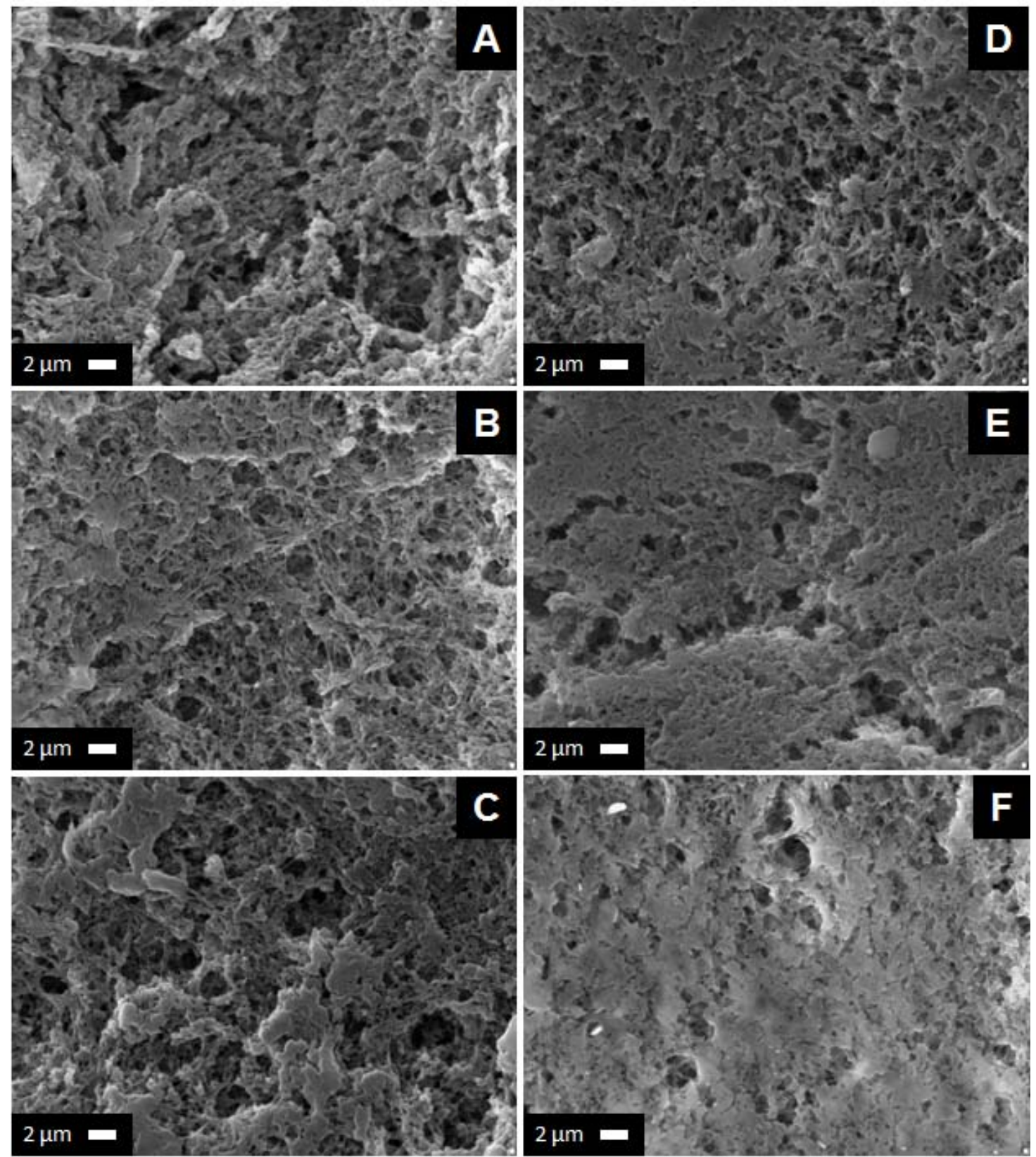

Figure 11. SEM images of PEGDA grafted microporous polypropylene membranes (A) $0 \%$ graft (B) $1.1 \%$ graft (C) $5.7 \%$ graft (D) $10.5 \%$ graft (E) $14.9 \%$ graft (F) $39.3 \%$ graft 
Figure 11 shows the SEM imagery of PEGDA $M_{n} 575$ grafted microporous polypropylene membranes with various grafting degrees. Image (A) depicts the untreated microporous polypropylene membrane after rinsing with acetone and exposure to the grafting solution excluding the PEGDA. With increasing grafting degrees from images A-F, an increase of grafted material can be seen on the surface. As more grafting material is shown, the visible surface pores of the membranes appear to decrease in size, or begin to be blocked by the surface grafted layer of PEGDA. Images A-D do not show a large blockage of these surface pores, as it is believed the majority of PEGDA surface grafting is occurring within the bulk pore structure of the substrate. Between images D and E, with a grafting degree around 12\% (not shown), it is believed that a fairly uniform layer of PEGDA hydrogel has been grafted to the membrane surface, and with any higher grafting degree this hydrogel layer is simply becoming thicker. This is confirmed in further detail by the FTIR analysis.

The aim of this current study was to create a moisture-responsive membrane where the swelling of the grafted PEGDA when exposed to moisture would cause the pore sizes of the membrane to decrease. Based on SEM imagery, it is believed that this moisture-responsive property is achievable with grafting degrees below 12\%. Images A-D depict the surface pore structures which should have this moisture-responsive property. Images E and F depict membranes which have a relatively thick layer of PEGDA hydrogel grafted on to the surface, blocking the surface pores and through pores. It is unlikely that the desired moisture-responsive property is achievable with such high grafting degrees as these, although there does appear to be some semblance of a pore structure visible on the hydrogel surface. 


\section{3-3. Fourier Transform Infrared Spectroscopy}

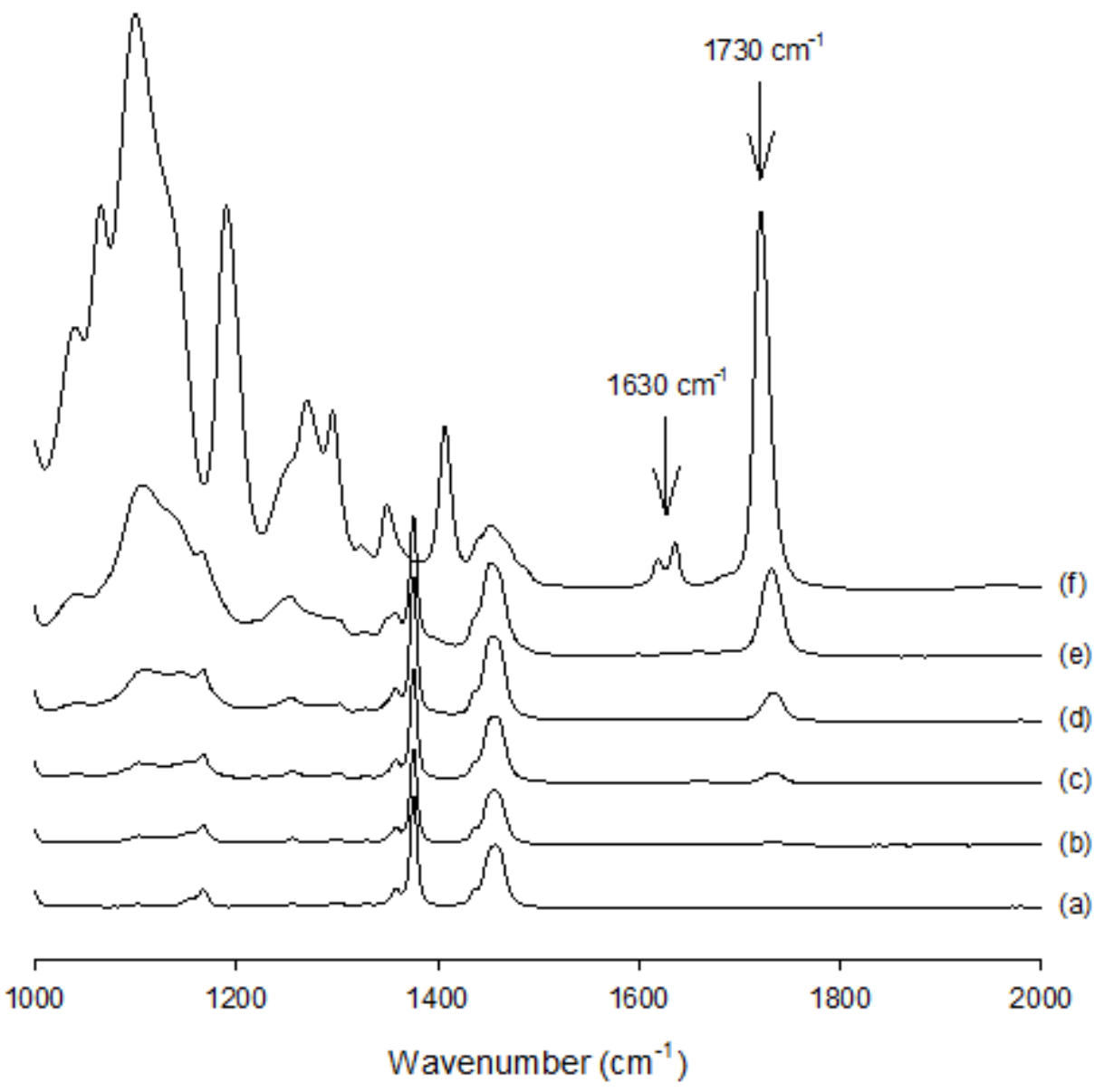

Figure 12. FTIR spectra of PEGDA grafted microporous polypropylene membranes (a) $0 \%$ graft (b) $1.1 \%$ graft $(c) 2.4 \%$ graft $(d) 9.7 \%$ graft (e) $12.3 \%$ graft (f) PEGDA $M_{n} 575$

Figure 12 shows the FTIR spectra of PEGDA $M_{n} 575$ grafted microporous polypropylene membranes with various grafting degrees as well as a spectra of PEGDA $M_{n} 575$ polymer (f). The spectra of PEGDA $M_{n} 575$ (f) is of the nascent polymer, not yet exposed to UV irradiation, therefore not cross-linked to form a hydrogel. This spectra shows the identifying peaks of PEGDA as described by previous published literature [25]. The carbonyl groups of the acrylate groups located 
on each end of the PEGDA chain is signified by the peak at $1730 \mathrm{~cm}^{-1}$. The peak at $1630 \mathrm{~cm}^{-1}$ signifies the carbon-carbon double bond located on each acrylate group of PEGDA which is available for cross-linking or grafting reactions. When PEGDA is exposed to UV light with a photoinitiator, the carbon-carbon double bonds are consumed in the cross-linking reaction and a hydrogel is formed; however, the carbonyl groups are still located within the structure. The spectra of a-e are of PEGDA grafted microporous polypropylene membranes with increasing grafting degrees. The carbonyl peak at $1730 \mathrm{~cm}^{-1}$ can be seen with an increasing strength as the grafting degree increases. The carbon-carbon double bond $1630 \mathrm{~cm}^{-1}$ peak is not visible on any of the grafted membrane samples since these bonds have been consumed in the crosslinking and grafting reactions. These spectra indicate that an increasing layer of PEGDA hydrogel is grafted on to the substrate surface with increasing grafting degree.

Although not shown in the figure, it was found that the carbonyl peak does not get significantly stronger with grafting degrees higher than $12.3 \%$, shown in spectra (e). This indicates that at about $12 \%$ graft, a relatively uniform layer of PEGDA hydrogel has been grafted to the substrate surface, and any additional PEGDA grafted to the substrate only results in a thicker layer of hydrogel bonded to the surface. As mentioned in the previous discussion on the SEM imagery, this indicates that the moisture-responsive properties desired by this study is likely achievable with grafting degrees less than $12 \%$. With grafting degrees higher than $12 \%$, the hydrogel layer on the substrate surface only manages to get thicker, no longer modifying the pore structure in the desired manner. The desired manner being a bulk porous structure with PEGDA grafted on the surface, decreasing the through pore sizes when in the non- 
swollen state. When exposed to moisture, the grafted PEGDA will swell and decrease the pore sizes further. Once moisture is removed, the PEGDA will contract back to the original non-swollen state, returning to the original decreased pore size.

\section{3-4. Contact Angle Measurements}

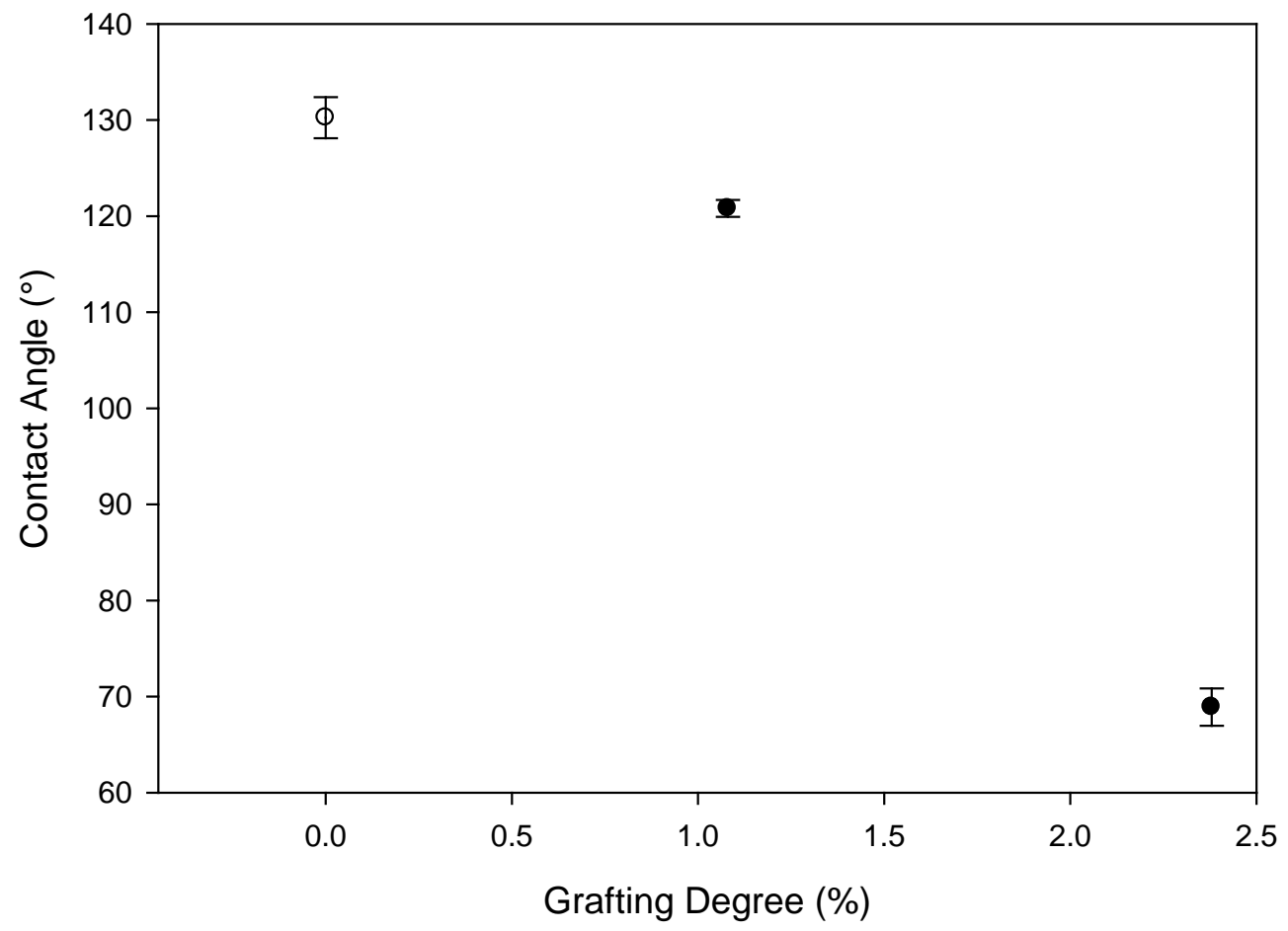

Figure 13. The effect of grafting degree on water contact angle of PEGDA grafted microporous polypropylene membranes

Figure 13 shows the effect of grafting degree on the water contact angle measurements of PEGDA $\mathrm{M}_{\mathrm{n}} 575$ grafted microporous polypropylene membranes. Only three grafting degrees were measured, as higher grafting degrees were found to be too hydrophilic for accurate water contact angle measurements to be made on the available instrument. A fairly exponential relationship can be seen between the grafting degree and water contact angle. With increasing grafting degree of PEGDA to 
the membrane surface, the water contact angle quickly decreases. A lower water contact angle indicates a higher hydrophilicity. This is as expected and further confirms successful grafting of PEGDA to the microporous polypropylene membrane surface. The nascent polypropylene membrane is relatively hydrophobic, with a high contact angle as shown in the plot at about $130^{\circ}$. When the PEGDA hydrogel, which is highly hydrophilic and swells in response to moisture, is grafted to the hydrophobic membrane surface, hydrophilicity is expected to increase. This increase in hydrophilicity is shown by the water contact angle measurements. With an increase in grafting degree, the water contact angles decrease significantly, indicating an increase in hydrophilicity due to the grafting of PEGDA to the membrane surface.

Modifying hydrophobic polypropylene in order to give it hydrophilic properties is a common modification done with a variety of methods [10, 29, 30]. This hydrophilic modification is often done in order to reduce the fouling which can occur with hydrophobic materials. Fouling refers to the build-up of material on the membrane surface, often protein, which reduces the effectiveness or flux of the membrane; this problem can be solved with a variety of methods, increasing hydrophilicity being one of them [31]. The data from this current study show that PEGDA is another suitable hydrophilic polymer which can be grafted to a hydrophobic membrane surface through UV-initiated grafting in order to increase the hydrophilic properties of the membrane, reducing the possible fouling. However, the aim of this current study is to create a moisture-responsive membrane using this grafting procedure, and the increased hydrophilicity and subsequent prevention of fouling is simply an extra benefit of the surface modification. 


\section{3-5. Porometry}

3-5-1. Air Flow Rate Comparisons of Various Grafting Degrees

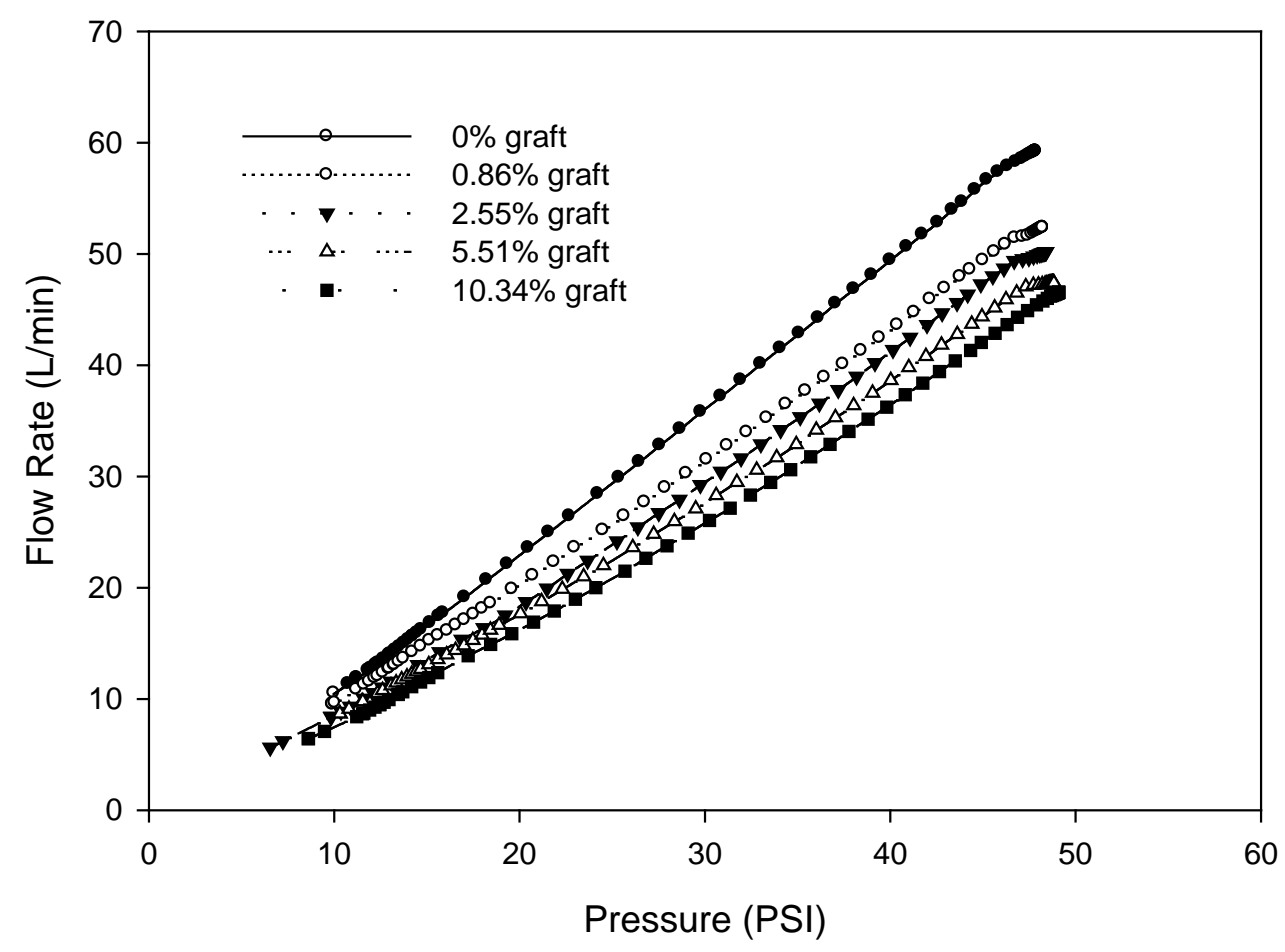

Figure 14. Dry air flow rate comparisons of PEGDA grafted microporous polypropylene membranes with various grafting degrees

Figure 14 shows the dry air flow rate comparisons of PEGDA $M_{n} 575$ grafted microporous polypropylene membranes with various grafting degrees. These data were taken from the porometry measurements made by forcing air with incrementally increasing pressure through the membrane and measuring the amount of air that passes through. The data shown are of the air flow rates through dry membranes. The air flow rates through membranes after saturation with a wetting liquid are not shown, as similar results were achieved. It is also unknown how the grafted PEGDA responds to the wetting liquid, as any swelling caused by this liquid may affect the results. This air flow rate comparison shows that with increasing grafting degree there is a decreasing 
air flow rate allowed through the membrane. This indicates that as more PEGDA is grafted to the substrate, the smaller the pore sizes become, allowing less air to pass through the membrane. It is also likely that the smallest pore sizes are being completely blocked by grafted PEGDA, preventing any air flow through them. These data confirm the predictions of this study that the initial grafting of PEGDA decreases the pore sizes of the microporous polypropylene membrane even when in the nonswollen state. The pore sizes are expected to decrease further when the membrane is exposed to moisture and the grafted PEGDA swells.

These air flow rate measurements were taken after the samples were vacuum dried in an attempt to remove all water content from the membranes. However, it appears to be quite difficult to completely remove all the water content from the grafted PEGDA hydrogel in an attempt to achieve a completely non-swollen state. This can be seen in the comparison of the air flow rates. There is a relatively large decrease in air flow rate from the untreated sample ( $0 \%$ graft) to the low grafting degree sample ( $0.86 \%$ graft). However, with further increase in grafting degree, the air flow rates decrease less substantially, although still significantly. This could indicate that the grafted samples are already in a slightly swollen state, as they could have absorbed moisture quickly from the air when attempting to measure the air flow rates. However, it is believed that the samples are not nearly at the completely swollen state, and will still exhibit moisture-responsive properties by a further decrease in pore sizes when exposed to moisture. 


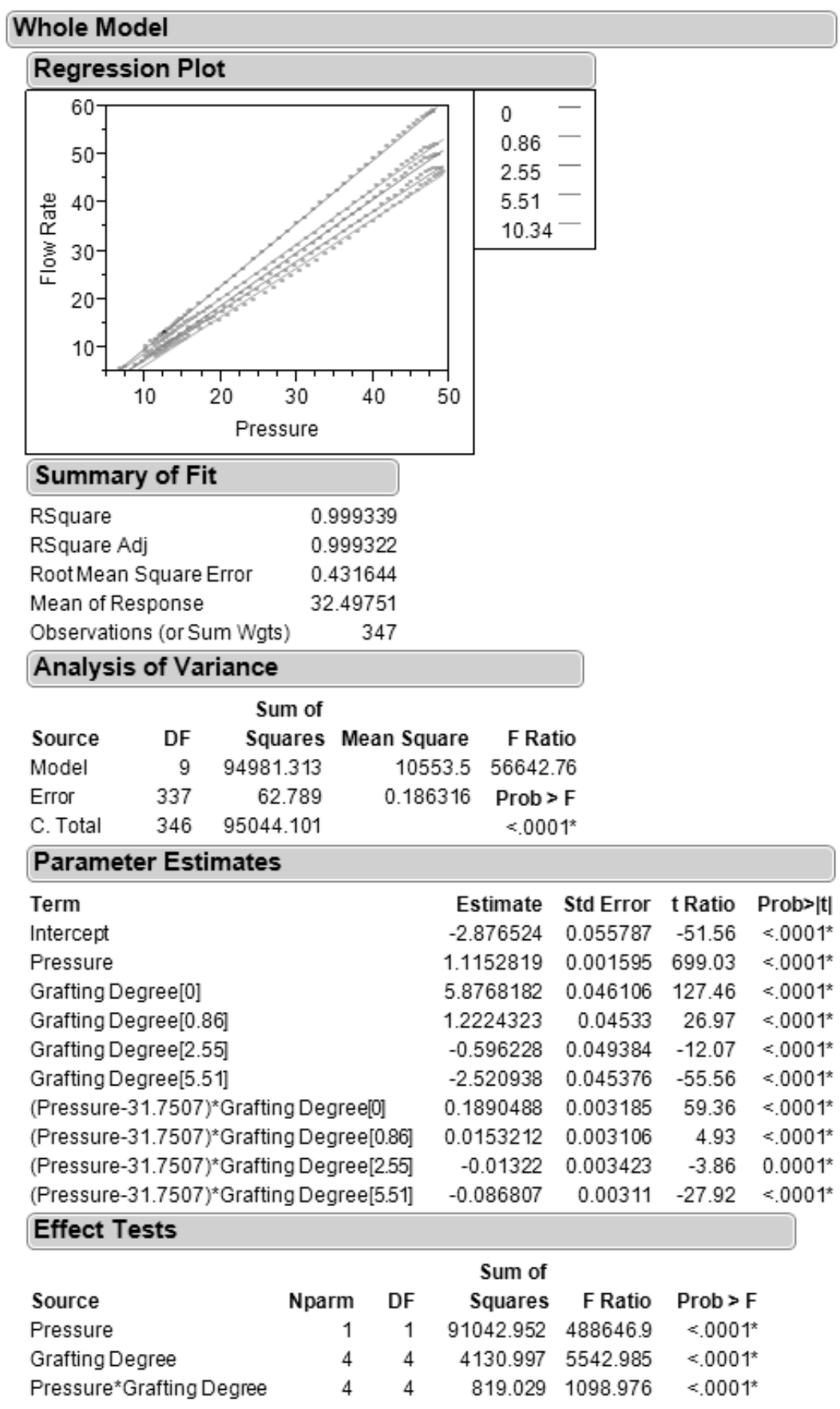

Figure 15. JMP statistical output for the ANCOVA of the dry air flow rate comparisons of membranes with various grafting degrees

Figure 15 shows the ANCOVA of the regression models for the five dry air

flow rate data sets of the PEGDA grafted microporous polypropylene membranes with 
various grafting degrees. Each regression line corresponds to the grafting degree listed in the legend to the right of the regression plot. ANCOVA was run on these data sets in order to determine if there was a statistically significant difference between the dry air flow rates of the samples with various grafting degrees. ANCOVA was used to determine if the five regression lines statistically differed in slope. In order to accomplish this, a dummy variable was used. This dummy variable is labeled as "Grafting Degree" in the output. The following model was fit to all of the data points from all five sets:

$$
\begin{aligned}
\text { Flow Rate }= & \beta_{0}+\beta_{1}(\text { Pressure })+\beta_{2}(\text { Grafting Degree }[0]) \\
& +\beta_{3}(\text { Pressure } \times \text { Grafting Degree }[0]) \\
& +\beta_{4}(\text { Grafting Degree }[0.86]) \\
& +\beta_{5}(\text { Pressure } \times \text { Grafting Degree }[0.86]) \\
& +\beta_{6}(\text { Grafting Degree }[2.55]) \\
& +\beta_{7}(\text { Pressure } \times \text { Grafting Degree }[2.55]) \\
& +\beta_{8}(\text { Grafting Degree }[5.51]) \\
& +\beta_{9}(\text { Pressure } \times \text { Grafting Degree }[5.51])+\varepsilon
\end{aligned}
$$

The hypothesis tested was $\mathrm{H}_{0}: \beta_{3}=\beta_{5}=\beta_{7}=\beta_{9}=0$ which hypothesizes that all five slopes are equal. The "Parameters Estimates" section shows that the parameter estimates for all Pressure*Grafting Degree variables are unequal to each other nor equal to $0\left(\beta_{3} \neq \beta_{5} \neq \beta_{7} \neq \beta_{9} \neq 0\right)$ with very small $\mathrm{p}$-values. This is enough evidence to reject the hypothesis and conclude that the slopes of the five regression lines are not equal. ANCOVA has shown that the differences between the five dry air flow rate data sets are statistically significant. This confirms that the initial grafting of PEGDA to the 
microporous polypropylene membrane surface has a statistically significant effect on the dry air flow rates, thus pore sizes, when in the non-swollen state. With increasing grafting degree of PEGDA to the substrate surface, there is a statistically significant decrease in dry air flow rate and pore sizes.

\section{3-5-2. Pore Size Distribution Comparisons of Various Grafting Degrees}

(a)

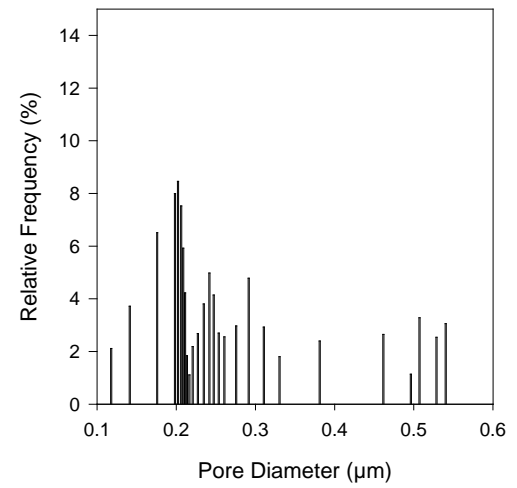

(b)

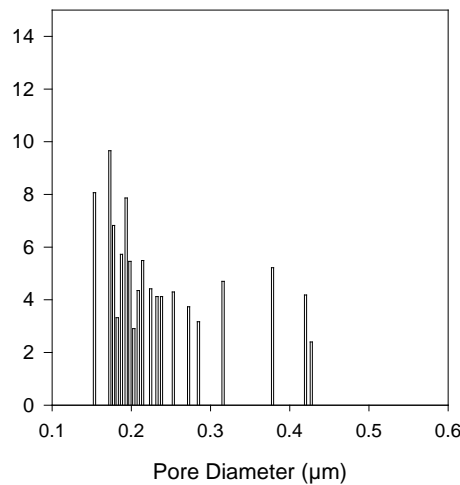

(c)

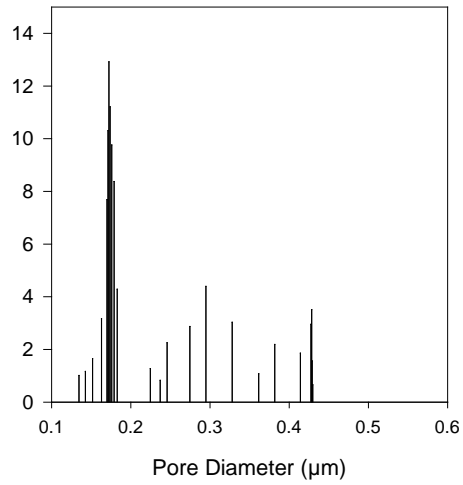

Figure 16. Pore size distributions of PEGDA grafted microporous polypropylene membranes with various grafting degrees (a) $0 \%$ graft (b) $1.31 \%$ graft (c) $14.47 \%$ graft

Figure 16 shows the pore size distributions of PEGDA grafted microporous polypropylene membranes with three different grafting degrees. These measurements were once again taken after samples were vacuum dried in an attempt to remove all moisture content from the grafted PEGDA, thus reducing the swelling. The pore size distributions shown are the relative frequencies of the pore diameters for each sample. Relative frequencies are used since the actual pore size counts have a high variability between samples with the same grafting degree, thus cannot give accurate comparisons of samples with different grafting degrees. However, the pore size counts 
can be indirectly compared through the dry air flow rates discussed previously, which has been shown to decrease with increasing grafting degree. This particular comparison of pore size distributions shows how the pore sizes are distributed within each sample.

The pore size distributions show that the larger pore diameters, $0.45 \mu \mathrm{m}$ and above, no longer appear for the two grafting degrees higher than $0 \%$ shown in (b) and (c). These two distributions also show higher relative frequencies for the smaller pore diameters when compared to the (a) distribution of $0 \%$ graft. The distribution (c) for the $14.47 \%$ graft sample shows the largest relative frequency at one of the smallest pore diameters, at about $0.16 \mu \mathrm{m}$. The distribution for this sample also shows very few pore sizes distributed at the pore diameters above $0.2 \mu \mathrm{m}$. The comparisons between these distributions indicate that the PEGDA grafted to the substrate surface is decreasing the larger pore sizes, creating a higher relative frequency of smaller pore sizes. This shows that the initial grafting of PEGDA to the surface of microporous polypropylene membranes decreases the pore sizes when in the non-swollen state. However, since these distributions are given in relative frequencies, the smaller pore sizes that have been filled up completely with grafted PEGDA are not accounted for. It is believed that these blocked pores are accounted for by the dry air flow rate measurements. 


\section{3-5-3. Air Flow Rate Comparison of Different Relative Humidity}

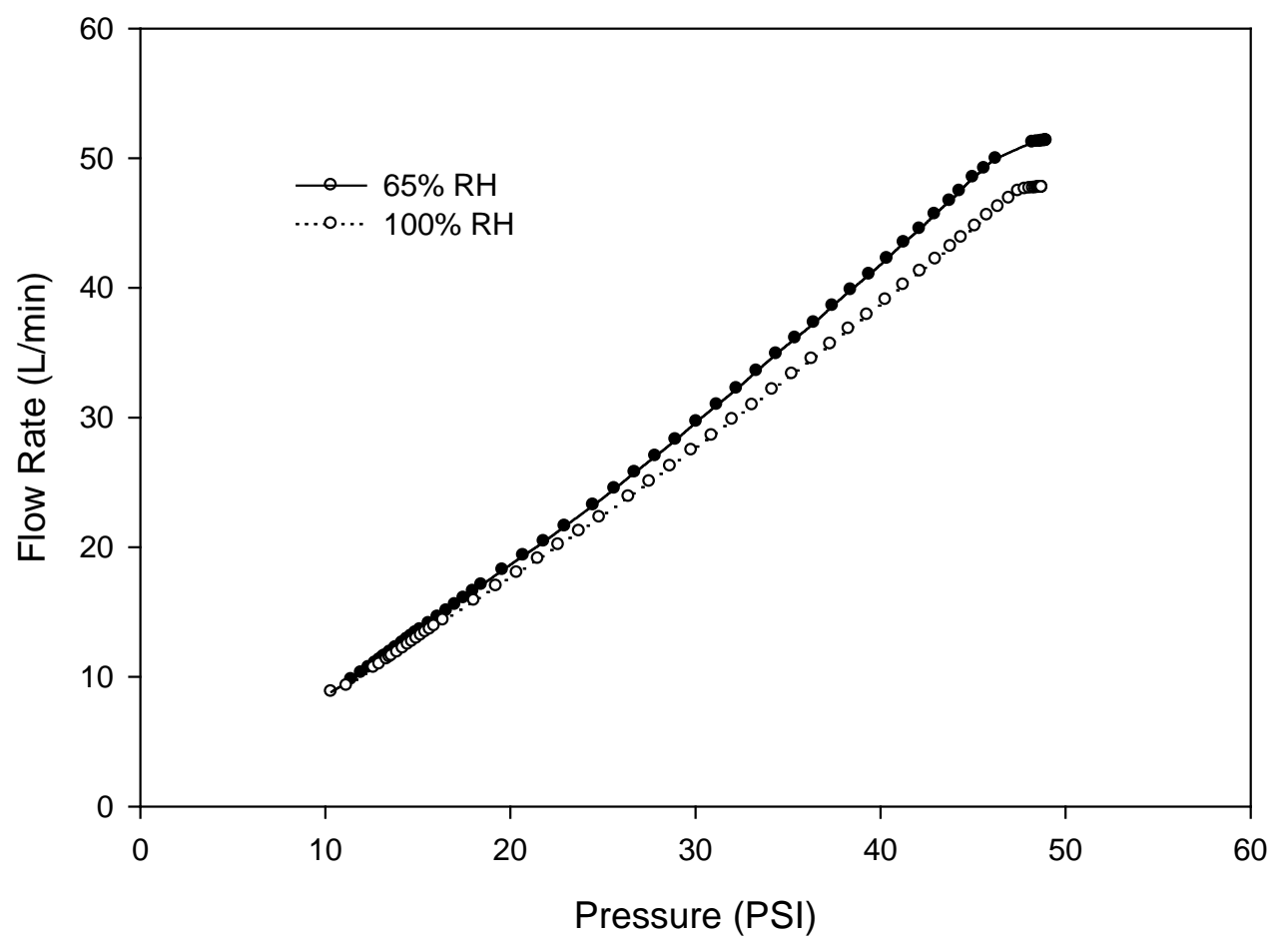

Figure 17. Dry air flow rate comparison of PEGDA grafted microporous polypropylene membranes conditioned at different relative humidity (RH)

Figure 17 shows the dry air flow rate comparison of PEGDA grafted microporous polypropylene membranes conditioned overnight at different relative humidity. Both samples had about a $4 \%$ grafting degree. The samples were conditioned overnight at $21^{\circ} \mathrm{C}$ with one conditioned at $65 \%$ relative humidity and the other conditioned at a theoretical $100 \%$ relative humidity. Porometry measurements were attempted on a sample conditioned at $0 \%$ relative humidity, but significant results were not found as it is believed that the membrane is able to quickly absorb moisture content from the air. The data shown are of the air flow rate measurements taken before the samples were saturated with a wetting liquid; this is referred to as the dry air flow rates. The results show that due to the difference in relative humidity, that 
is the available moisture content in the air, the samples exhibit a difference in air flow rates. With a higher relative humidity, the dry air flow rate is significantly lower. This indicates that the desired moisture-responsive properties have been achieved: with the higher available moisture content in the air, the grafted PEGDA swells and results in a reduced air flow rate. This reduced air flow rate indicates a reduction in pore sizes.

The pore sizes are expected to decrease even further when exposed to higher water content, which could be through contact with a liquid-borne pathogen. The desired moisture-responsive membrane for this study is expected to uptake moisture content from the air, or from exposure to a liquid-borne pathogen, and the grafted PEGDA is expected to swell in response, reducing the pore sizes and enhancing protection. The hope is to create a membrane for protective applications which can provide enhanced protection without completely sacrificing comfort. The comfort is provided by the microporous structure which allows water vapor to pass through the membrane. This dry air flow rate comparison confirms that the desired moistureresponsive property has been achieved through UV-initiated grafting of PEGDA to the surface of microporous polypropylene membranes. With the increase in relative humidity, the membrane responds with a reduction in pore sizes. The grafted PEGDA is expected to be able to swell to an even greater degree when exposed to a higher moisture content, decreasing the pore sizes even further. 


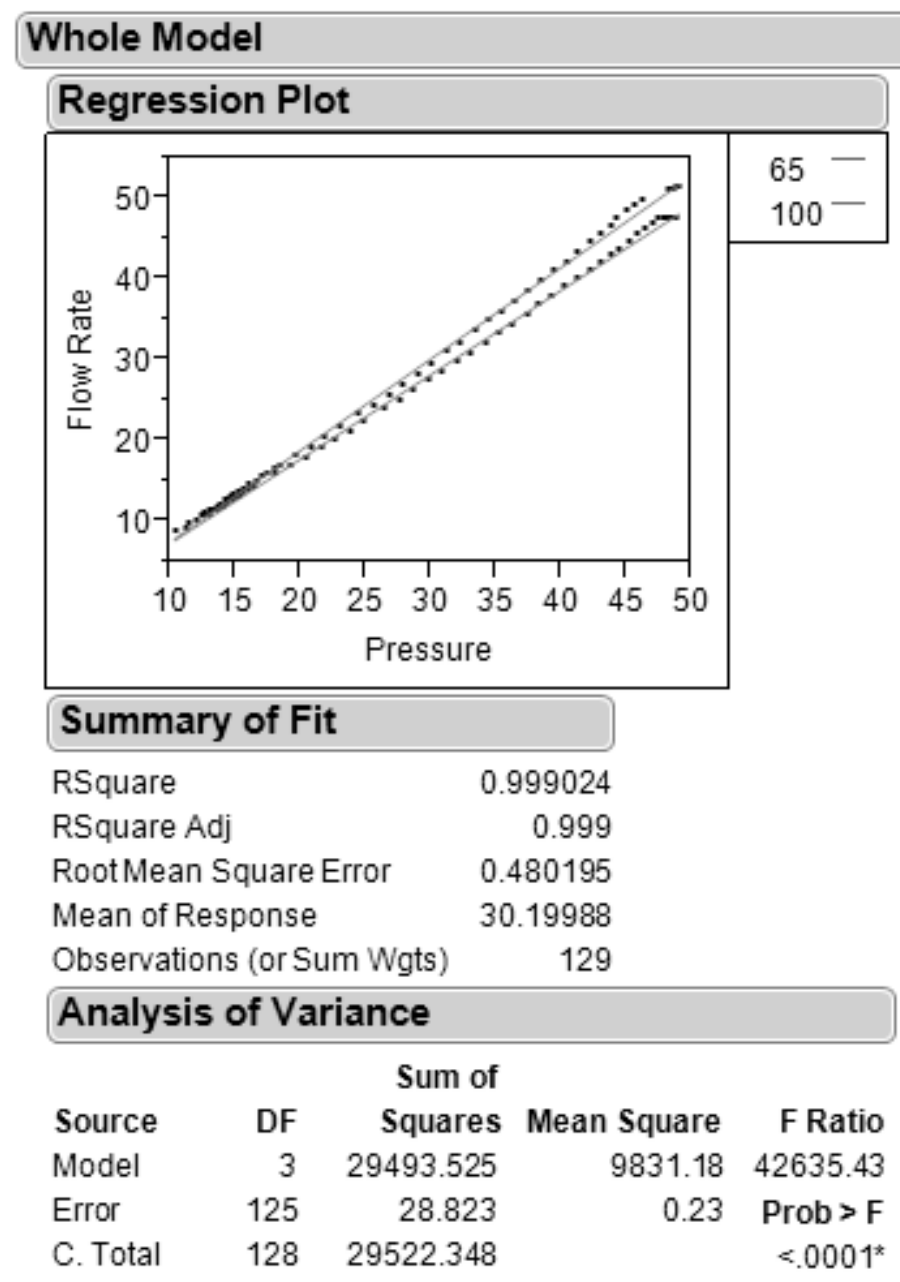

\section{Parameter Estimates}

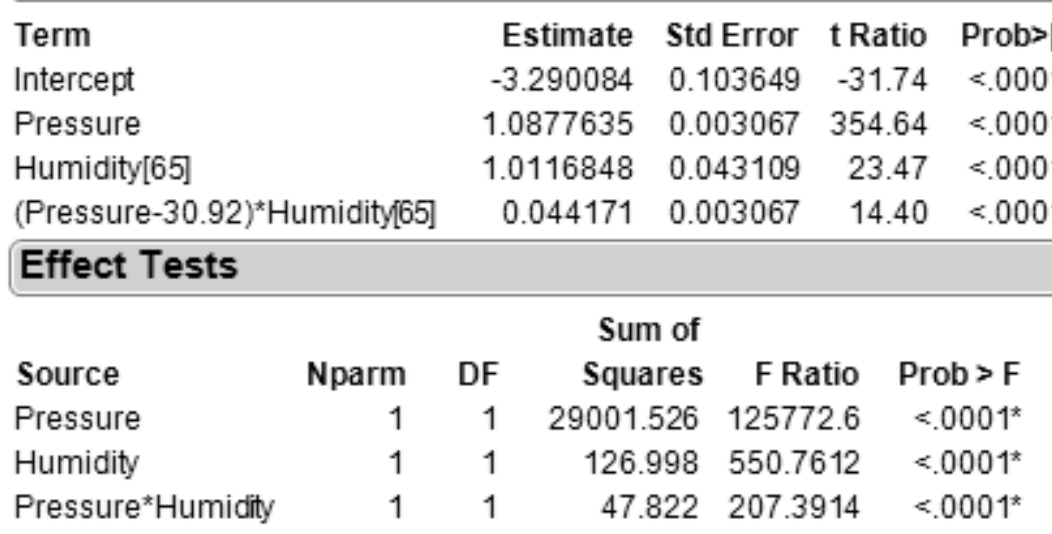

Figure 18. JMP statistical output for the ANCOVA of the dry air flow rate comparison of grafted membranes conditioned at different relative humidity 
Figure 18 shows the ANCOVA of the regression models for the two dry air flow rate data sets of the PEGDA grafted microporous polypropylene membranes conditioned at different relative humidity. The top regression line on the regression plot corresponds to the sample conditioned at $65 \%$ relative humidity, while the bottom line corresponds to the $100 \%$ relative humidity sample. ANCOVA was run on these data sets in order to determine if there was a statistically significant difference between the dry air flow rates of the samples conditioned at different relative humidity. This can be used to confirm that the desired moisture-responsive swelling property has indeed been achieved. Essentially, ANCOVA was used to determine if the two regression lines statistically differed in slope. In order to accomplish this, a dummy variable was used. This dummy variable is labeled as "Humidity" in the output. The following model was fit to all of the data points from both sets:

$$
\begin{aligned}
\text { Flow Rate }= & \beta_{0}+\beta_{1}(\text { Pressure })+\beta_{2}(\text { Humidity })+\beta_{3}(\text { Pressure } \times \text { Humidity }) \\
& +\varepsilon
\end{aligned}
$$

The hypothesis tested was $\mathrm{H}_{0}: \beta_{3}=0$ which hypothesizes that both slopes are equal. The "Parameters Estimates" section shows that the parameter estimate for Pressure*Humidity is not equal to $0\left(\beta_{3} \neq 0\right)$ with a very small $p$-value. This is enough evidence to reject the hypothesis and conclude that the slopes of the two regression lines are not equal. ANCOVA has shown that the difference between the two dry air flow rate data sets is statistically significant. This assists in confirming that the desired moisture-responsive property has been achieved. With an increase in relative humidity, the air flow rate through the PEGDA grafted membrane is decreased in a 
statistically significant amount. This indicates that the pore sizes of the membrane decrease significantly in response to moisture exposure. 


\section{CHAPTER 4}

\section{CONCLUSIONS}

This study has successfully grafted PEGDA to the surface of a microporous polypropylene membrane through UV-initiated grafting. This surface modification has been shown to improve hydrophilicity of the nascent hydrophobic polypropylene membrane through water contact angle measurements. The grafting conditions studied, grafting solution concentration, PEGDA molecular weight, and UV exposure time, have been shown to greatly affect the grafting degree of PEGDA to the substrate surface. These conditions can be controlled in order to create a grafted membrane with the desired grafting degree as well as swelling property.

Porometry has shown that the initial graft of PEGDA to the substrate surface decreases the pore sizes when in the non-swollen state. The amount of pore size decrease is dependent on the grafting degree. The grafted PEGDA has also been shown to respond to moisture, decreasing pore sizes further when exposed to moisture, through a difference in relative humidity, which causes the grafted polymer to swell. This moisture-responsive property can enhance protection of the membrane when exposed to possible liquid-borne pathogens. 


\section{4-1. Recommendations for Future Studies}

\section{4-1-1. Water Vapor Transmission Rate}

This current study has only shown that the PEGDA grafted microporous polypropylene membrane has moisture-responsive properties. However, it has yet to quantify this property. There is an instrument which can help to quantify this called the Water Vapor Transmission Analyzer (Porous Materials Inc., Ithaca, NY) [32]. This analyzer is able to measure the water vapor transmission rate (WVTR) of a sample while creating any desired temperature, pressure, or humidity gradient. This is done using a similar method to porometry. However, instead of forcing air with just incrementally increasing pressure through the sample, the humidity of the air is controlled. The temperature of the sample chamber is also controlled. This can provide a very detailed characterization of the moisture-responsive properties of the membrane fabricated in this study. The amount of moisture content passing through the membrane can be controlled, and the changes in WVTR and pore structure properties in response to the humidity gradient can be measured. Originally, this study had plans to characterize the fabricated membranes with this method. Unfortunately, the instrument is currently unavailable. However, once there is one available, it would be very interesting to further characterize the moisture-responsive properties of PEGDA grafted microporous polypropylene membranes. 


\section{4-1-2. Artificial Leaf Stomata}

An idea that was considered for this current study, provided by Professor Jin-tu Fan originally from The Hong Kong Polytechnic University, now at Cornell University, was to create a porous structure that would mimic a leaf stomata. Leaf stomata are the natural pores located on a leaf which essentially help it to breathe. They are known to open and close in response to light, but also in response to moisture, or changes in humidity [33]. The artificially created pores would focus on this change in response to moisture. However, unlike the membrane fabricated in this current study, the pore sizes would increase in response to moisture, rather than decrease. Instead of protective applications, this type of moisture-responsive membrane would be more focused on comfort. When the user begins to heat up and produce sweat, the pore sizes of the membrane would increase, allowing more water vapor to be transported away from the body. Once the users cools down, the pore sizes would return to their original smaller size, decreasing the air permeability. This moisture-responsive structure would help regulate the user's body temperature. This type of moisture-responsive membrane could be created using similar materials as this current study. The swelling properties of PEGDA could be utilized in such a way that would physically open up a pore. This can be done with some clever selective grafting using photolithography. A similar concept has already been demonstrated where flat polymer sheets swell and buckle in response to stimuli such as temperature. These flat sheets transform in to three-dimensional shapes when swollen, and can revert back to a flat sheet once the stimuli is removed [34]. 


\section{REFERENCES}

[1] M. Mänttäri, L. Puro, J. Nuortila-Jokinen and M. Nyström, "Fouling effects of polysaccharides and humic acid in nanofiltration," Journal of Membrane Science, vol. 165, pp. 1-17, 1/17, 2000.

[2] L. Lange and S. Obendorf, "Effect of Plasma Etching on Destructive Adsorption Properties of Polypropylene Fibers Containing Magnesium Oxide Nanoparticles," Archives of Environmental Contamination and Toxicology, vol. 62, pp. 185-194, 2012.

[3] V. Dixit, J. Tewari and S. Obendorf, "Fungal Growth Inhibition of Regenerated Cellulose Nanofibrous Membranes Containing Quillaja Saponin," Archives of Environmental Contamination and Toxicology, vol. 59, pp. 417-423, 2010.

[4] S. Lee and S. Kay Obendorf, "Developing protective textile materials as barriers to liquid penetration using melt-electrospinning," Journal of Applied Polymer Science, vol. 102, pp. 3430-3437, 2006.

[5] C. N. Nelson, N. W. Henry and Clothing,ASTM Committee F-23 on Protective, Performance of Protective Clothing: Issues and Priorities for the 21st Century. Seventh Volume. ASTM, 2000.

[6] J. M. Harris, Poly(Ethylene Glycol) Chemistry: Biotechnical and Biomedical Applications. New York: Plenum Press, 1992.

[7] N. C. Padmavathi and P. R. Chatterji, "Structural Characteristics and Swelling Behavior of Poly(ethylene glycol) Diacrylate Hydrogels ," Macromolecules, vol. 29, pp. 1976-1979, 01/01; 2012/04, 1996.

[8] H. Kim, H. Kim, C. Hou and B. Kim, "Biodegradable Photo-Crosslinked Thin Polymer Networks Based on Vegetable Oil Hydroxy Fatty Acids," JAOCS: Journal of the American Oil Chemists' Society, vol. 87, pp. 1451-1459, 12, 2010.

[9] C. Tu, C. Chen, Y. Wang, C. Li, H. Tsai, K. Lee and J. Lai, "Acrylamide plasmainduced polymerization onto expanded poly(tetrafluoroethylene) membrane for aqueous alcohol mixture vapor permeation separation," European Polymer Journal, vol. 40, pp. 1541-1549, 7, 2004.

[10] Y. Wang, J. Kim, K. Choo, Y. Lee and C. Lee, "Hydrophilic modification of polypropylene microfiltration membranes by ozone-induced graft polymerization," Journal of Membrane Science, vol. 169, pp. 269-276, 5/1, 2000.

[11] D. S. Wavhal and E. R. Fisher, "Membrane Surface Modification by PlasmaInduced Polymerization of Acrylamide for Improved Surface Properties and Reduced Protein Fouling," Langmuir, vol. 19, pp. 79-85, 01/01; 2011/11, 2003.

[12] K. Kato, E. Uchida, E. Kang, Y. Uyama and Y. Ikada, "Polymer surface with graft chains," Progress in Polymer Science, vol. 28, pp. 209-259, 2, 2003.

[13] M. Nyström and P. Järvinen, "Modification of polysulfone ultrafiltration membranes with UV irradiation and hydrophilicity increasing agents," Journal of Membrane Science, vol. 60, pp. 275-296, 1987.

[14] M. Ebara, J. M. Hoffman, P. S. Stayton and A. S. Hoffman, "Surface modification of microfluidic channels by UV-mediated graft polymerization of 
non-fouling and 'smart' polymers," Radiation Physics and Chemistry, vol. 76, pp. 1409-1413, 9, 2007.

[15] K. T. Nguyen and J. L. West, "Photopolymerizable hydrogels for tissue engineering applications," Biomaterials, vol. 23, pp. 4307-4314, 11, 2002.

[16] B. Rånby, W. T. Yang and O. Tretinnikov, "Surface photografting of polymer fibers, films and sheets," Nuclear Instruments and Methods in Physics Research Section B: Beam Interactions with Materials and Atoms, vol. 151, pp. 301-305, 5/2, 1999.

[17] C. Oehr, M. Müller, B. Elkin, D. Hegemann and U. Vohrer, "Plasma grafting — a method to obtain monofunctional surfaces," Surface and Coatings Technology, vol. 116-119, pp. 25-35, 9, 1999.

[18] K. Tan and S. K. Obendorf, "Surface modification of microporous polyurethane membrane with poly(ethylene glycol) to develop a novel membrane," Journal of Membrane Science, vol. 274, pp. 150-158, 04/05, 2006.

[19] Z. Shaofeng, "Surface Modification of Polypropylene Microporous Membrane by Atmospheric-Pressure Plasma Immobilization of N,N-dimethylamino Ethyl Methacrylate," Plasma Science and Technology, vol. 12, pp. 619, 2010.

[20] Q. Yang, Z. Xu, Z. Dai, J. Wang and M. Ulbricht, "Surface Modification of Polypropylene Microporous Membranes with a Novel Glycopolymer," Chemistry of Materials, vol. 17, pp. 3050-3058, 05/01, 2005.

[21] W. Li, P. Liu, H. Zou, P. Fan and W. Xu, "pH sensitive microporous polypropylene membrane prepared through ozone induced surface grafting," Polymers for Advanced Technologies, vol. 20, pp. 251-257, 2009.

[22] H. Iwata, M. I. Ivanchenko and Y. Miyaki, "Preparation of anti-oil stained membrane by grafting polyethylene glycol macromer onto polysulfone membrane," Journal of Applied Polymer Science, vol. 54, pp. 125-128, 1994.

[23] Y. G. Ko, Y. H. Kim, K. D. Park, H. J. Lee, W. K. Lee, H. D. Park, S. H. Kim, G. S. Lee and D. J. Ahn, "Immobilization of poly(ethylene glycol) or its sulfonate onto polymer surfaces by ozone oxidation," Biomaterials, vol. 22, pp. 2115-2123, 8/1, 2001.

[24] S. Sugiura, J. Edahiro, K. Sumaru and T. Kanamori, "Surface modification of polydimethylsiloxane with photo-grafted poly(ethylene glycol) for micropatterned protein adsorption and cell adhesion," Colloids and Surfaces B: Biointerfaces, vol. 63, pp. 301-305, 6/1, 2008.

[25] D. Choi, W. Lee, J. Park and W. Koh, "Preparation of poly(ethylene glycol) hydrogels with different network structures for the application of enzyme immobilization," Bio-Medical Materials \& Engineering, vol. 18, pp. 345-356, 12 , 2008.

[26] T. R. Dargaville, G. A. George, D. J. T. Hill and A. K. Whittaker, "High energy radiation grafting of fluoropolymers," Progress in Polymer Science, vol. 28, pp. 1355-1376, 9, 2003.

[27] A. Jena and K. Gupta, "Advances in Pore Structure Evaluation by Porometry," Chemical Engineering \& Technology, vol. 33, pp. 1241-1250, 2010. 
[28] A. Tidjani, "Photooxidation of polypropylene under natural and accelerated weathering conditions," Journal of Applied Polymer Science, vol. 64, pp. 24972503, 1997.

[29] N. Inagaki, S. Tasaka and Y. Horikawa, "Durable, hydrophilic surface modification of polypropylene films by plasma graft polymerization of glycidyl methacrylate," Polymer Bulletin, vol. 26, pp. 283-289, 1991.

[30] S. Knaus and A. Nennadal, "Surface modification of polypropylene: Hydrophilic finishing with carbohydrates," Macromolecular Symposia, vol. 127, pp. 257-263, 1998.

[31] G. Belfort, R. H. Davis and A. L. Zydney, "The behavior of suspensions and macromolecular solutions in crossflow microfiltration," Journal of Membrane Science, vol. 96, pp. 1-58, 11/28, 1994.

[32] A. Jena, K. Gupta and M. Connolly, "Characterization of Water Vapor Transmission Rate Through Fuel Cell Components," ASME Conference Proceedings, vol. 2004, pp. 15-17, 01/01, 2004.

[33] P. J. Kramer and J. S. Boyer, Water Relations of Plants and Soils. Academic Press, 1995.

[34] J. Kim, J. A. Hanna, M. Byun, C. D. Santangelo and R. C. Hayward, "Designing Responsive Buckled Surfaces by Halftone Gel Lithography," Science, vol. 335, pp. 1201-1205, March 09, 2012. 